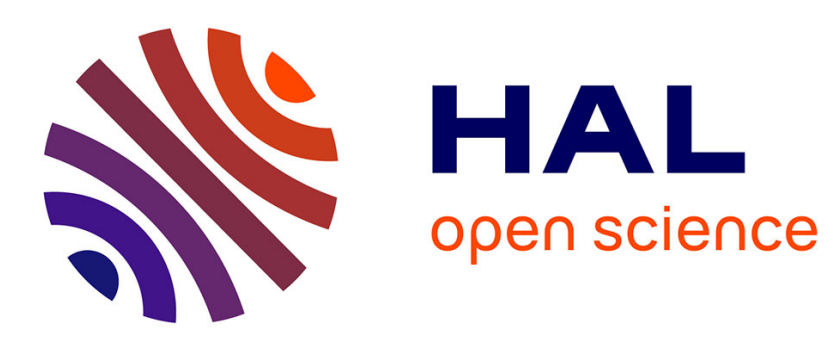

\title{
Quasi-local multi-trace boundary integral formulations
}

Xavier Claeys

\section{To cite this version:}

Xavier Claeys. Quasi-local multi-trace boundary integral formulations. Numerical Methods for Partial Differential Equations, 2015, 31 (6), pp.2043-2062. hal-01070264

\section{HAL Id: hal-01070264 \\ https://hal.science/hal-01070264}

Submitted on 2 Oct 2014

HAL is a multi-disciplinary open access archive for the deposit and dissemination of scientific research documents, whether they are published or not. The documents may come from teaching and research institutions in France or abroad, or from public or private research centers.
L'archive ouverte pluridisciplinaire HAL, est destinée au dépôt et à la diffusion de documents scientifiques de niveau recherche, publiés ou non, émanant des établissements d'enseignement et de recherche français ou étrangers, des laboratoires publics ou privés. 


\title{
Quasi-local multi-trace boundary integral formulations
}

\author{
X.Claeys ${ }^{* \dagger \ddagger}$
}

\begin{abstract}
In the context of time harmonic wave scattering by piecewise homogeneous penetrable objects, we present a new variant of the multi-trace boundary integral formulation, that differs from the local multi-trace approach of [Jerez-Hanckes \& Hiptmair, 2012] by the presence of regularisation terms involving boundary integral operators and localised at junctions i.e. points where at least three subdomains abut. We prove well-posedness and quasi-optimal convergence of conforming Galerkin discretisations for this new formulation, and present numerical results.
\end{abstract}

\section{Introduction}

Due to its potentially large scope of application, the analysis of boundary integral formulation for wave scattering by piecewise homogeneous objects has received a lot of attention in past decades. Several approaches, such as PMCHWT [20, 3, 15, 23, 2] or the BETI method $[18,21,22]$, have been derived rather early for treating scattering by geometrically complex structures by means of boundary integral operators. However, the existing approaches did not lend themselves (at least as local solvers) to some of the most popular preconditioning techniques such as Calderón's preconditioner [4, 5, 26].

This was the main motivation for the recent development of the so-called multi-trace formulations that were prone to Calderón preconditioning and, at the same time, adapted to boundary integral formulation of wave propagation in piecewise homogeneous media admitting points where three or more subdomains could be adjacent to each other. The so-called local multi-trace formulation, first proposed in [17], consists in writing wave equations separately in each subdomain of the propagation medium by means integral operators, and to couple subdomain together by means of a local transmission operator coming into play via a relaxation term of the equation. This formulation has been dubbed local because no integral operator is required to enforce transmission conditions.

As regards effective numerical performance, this formulation looks appealing. But few theory could be established. This is mostly due to the transmission operator that acts on Sobolev trace spaces, and involves at the same time restrictions and extensions by zero which are not continuous operations in natural trace norms. So far this formulation has been proved to admit a unique solution for general multi-dimensional cases, but no Garding's inequality

\footnotetext{
*Sorbonne Universités, UPMC Univ Paris 06, UMR 7598, Laboratoire Jacques-Louis Lions, Paris,

${ }^{\dagger}$ CNRS, UMR 7598, Laboratoire Jacques-Louis Lions, Paris,

${ }^{\ddagger}$ INRIA-Paris-Rocquencourt, EPC Alpines, Domaine de Voluceau, Le Chesnay.
} 
has been established, and discrete inf-sup conditions for conformal discretisations of this formulation has been studied only in a 2-D case.

In the present article we introduce a regularised version of the local multi-trace formulation. The regularisation terms are localised at the junction points, and this new variant of the multi-trace formulation of [17] lends itself to much more standard analysis: all operators are continuous in standard Sobolev trace spaces, a Garding inequality can be proved, which implies quasi-optimal approximation property for conformal Galerkin discretisations. As regards numerical performances, this new formulation also appears slightly more accurate compared to the original local multi-trace formulation. On the other hand, iterative solvers may be a little bit slower.

The outline of this article is as follows. In section 1 and 2 , we set some notations and describe precisely the problem under consideration: a standard scalar wave scattering by an arrangement of homogeneous objects. In section 3 and 4 we recall well established results of potential theory and provide a brief review of the derivation of the local multi-trace formulation considered in [17]. In Section 5 we introduce a new way of enforcing transmission conditions and, in Section 6, we deduce from this a regularised local multi-trace formulation. In the last section we present numerical results. We conclude in Section 7 with numerical experiments.

\section{Setting of the problem}

We first present in detail the problem that we will consider in this document. For $d=$ $1,2,3, \ldots$, consider a partition of the free space as $\mathbb{R}^{d}:=\cup_{j=0}^{n} \bar{\Omega}_{j}$ where the $\Omega_{j}$ 's are Lipschitz domains. We assume that each $\Omega_{j}$ is bounded except $\Omega_{0}$. In the sequel we shall refer to the boundary of each subdomain by $\Gamma_{j}:=\partial \Omega_{j}$, and also set $\Gamma_{j, k}:=\Gamma_{j} \cap \Gamma_{k}=\partial \Omega_{j} \cap \partial \Omega_{k}$ for reference to interfaces. The union of all interfaces will be denoted

$$
\Sigma:=\bigcup_{j=0}^{n} \Gamma_{j}=\bigcup_{0 \leq j<k \leq n} \Gamma_{j, k} .
$$

Following the standard terminology of domain decomposition, this set will be called the skeleton of the partition. As regards function spaces we follow the usual notations; given some open subset $\omega \subset \mathbb{R}^{d}$, we define $\mathrm{H}^{1}(\omega):=\left\{v \in \mathrm{L}^{2}(\omega) \mid \nabla v \in \mathrm{L}^{2}(\omega)\right\}$ with $\|v\|_{\mathrm{L}^{2}(\omega)}^{2}:=$ $\|v\|_{\mathrm{L}^{2}(\omega)}^{2}+\|\nabla v\|_{\mathrm{L}^{2}(\omega)}^{2}$, and $\mathrm{H}^{1}(\Delta, \omega):=\left\{v \in \mathrm{H}^{1}(\omega) \mid \Delta v \in \mathrm{L}^{2}(\omega)\right\}$. If $\mathrm{H}(\omega)$ is any one of these spaces, $\mathrm{H}_{\mathrm{loc}}(\bar{\omega}):=\left\{v \mid \varphi v \in \mathrm{H}(\omega) \forall \varphi \in \mathscr{C}_{\mathrm{K}}^{\infty}\left(\mathbb{R}^{d}\right)\right\}$, where $\mathscr{C}_{\mathrm{K}}^{\infty}\left(\mathbb{R}^{d}\right)$ refers to the space of $C^{\infty}$ function with compact support.

We consider a very standard wave scattering problem (so-called transmission problem), imposing Hemlholtz equation in each subdomain, as well as transmission conditions across interfaces: find $u \in \mathrm{H}_{\mathrm{loc}}^{1}\left(\mathbb{R}^{d}\right)$ such that

$$
\left\{\begin{array}{l}
-\Delta u-\kappa_{j}^{2} u=0 \text { in } \Omega_{j} \quad \forall j=0 \ldots n \\
u-u_{\text {inc }} \text { is } \kappa_{0} \text {-outgoing in } \Omega_{0} \\
\left.u\right|_{\Gamma_{j}}-\left.u\right|_{\Gamma_{k}}=0 \\
\left.\partial_{n_{j}} u\right|_{\Gamma_{j}}+\left.\partial_{n_{k}} u\right|_{\Gamma_{k}}=0 \text { on } \Gamma_{j, k}=\Gamma_{j} \cap \Gamma_{k}, \forall j, k=0 \ldots n
\end{array}\right.
$$


where $\partial_{n_{j}}$ refers to the outgoing normal derivative on $\partial \Omega_{j}$. In the equation above $u_{\text {inc }} \in$ $\mathrm{H}_{\text {loc }}^{1}\left(\mathbb{R}^{d}\right)$ is a known source term of the problem satisfying $-\Delta u_{\text {inc }}-\kappa_{0}^{2} u_{\text {inc }}=0$ in $\mathbb{R}^{d}$. In addition, we assume that $\kappa_{j}>0$ for all $j=0 \ldots n$. The outgoing radiation condition refers to Sommerfeld's condition i.e. we say that $v$ is $\kappa$-outgoing if

$$
\lim _{r \rightarrow \infty} \int_{\partial \mathrm{B}_{r}}\left|\partial_{r} v-\boldsymbol{\imath} \kappa v\right|^{2} d \sigma_{r}=0
$$

where $\mathrm{B}_{r}$ is the ball of centre 0 and radius $r$, and $\partial_{r}$ is the radial derivative. Problem (1) is known to admit a unique solution, see [23] for example.

As this problem involves transmission conditions, and since we are interested in boundary integral formulations of it, we need to introduce corresponding trace spaces and trace operators. Given any Lipschitz open subset $\omega \subset \mathbb{R}^{d}$ with bounded boundary, recall (see e.g. [25, Thm. 2.6.8]) that the map $\left.\varphi \mapsto \varphi\right|_{\partial \omega}$ can be extended as a continuous map from $\mathrm{H}_{\mathrm{loc}}^{1}(\omega)$ to $\mathrm{L}^{2}(\partial \omega)$. The set of Dirichlet traces on $\partial \omega$, denoted $\mathrm{H}^{1 / 2}(\partial \omega)$, is the range of this map. It is a Banach space when equipped with the norm $\|v\|_{\mathrm{H}^{1 / 2}(\partial \omega)}:=\inf \left\{\|\varphi\|_{\mathrm{H}^{1}(\omega)}, \varphi \in \mathrm{H}^{1}(\omega)\right.$ and $\left.\left.\varphi\right|_{\partial \omega}=v\right\}$. The set of Neumann traces on $\partial \omega$, denoted $\mathrm{H}^{-1 / 2}(\partial \omega)$, is the topological dual to this space, equipped with the canonical dual norm

$$
\|q\|_{\mathrm{H}^{-1 / 2}(\partial \omega)}:=\sup _{v \in \mathrm{H}^{1 / 2}(\partial \omega)} \frac{|\langle q, v\rangle|}{\|v\|_{\mathrm{H}^{1 / 2}(\partial \omega)}} .
$$

Recall also (see e.g. [25, Thm 2.7.7]) that the normal derivative $\left.\varphi \mapsto \mathbf{n} \cdot \nabla \varphi\right|_{\partial \omega}$ can be extended by continuity as an operator mapping $\mathrm{H}_{\mathrm{loc}}^{1}(\Delta, \bar{\omega})$ onto $\mathrm{H}^{-1 / 2}(\partial \omega)$.

As an application of the preceding remarks, every subdomain $\Omega_{j}$ gives rise to continuous boundary trace operators $\gamma_{\mathrm{D}}^{j}: \mathrm{H}_{\mathrm{loc}}^{1}\left(\bar{\Omega}_{j}\right) \rightarrow \mathrm{H}^{1 / 2}\left(\partial \Omega_{j}\right)$ and $\gamma_{\mathrm{N}}^{j}: \mathrm{H}_{\mathrm{loc}}^{1}\left(\Delta, \bar{\Omega}_{j}\right) \rightarrow \mathrm{H}^{-1 / 2}\left(\partial \Omega_{j}\right)$ (so-called Dirichlet and Neumann traces) uniquely defined by

$$
\gamma_{\mathrm{D}}^{j}(\varphi):=\left.\varphi\right|_{\partial \Omega_{j}} \quad \text { and } \quad \gamma_{\mathrm{N}}^{j}(\varphi):=\left.\mathbf{n}_{j} \cdot \nabla \varphi\right|_{\partial \Omega_{j}} \quad \forall \varphi \in \mathscr{C}^{\infty}\left(\bar{\Omega}_{j}\right) .
$$

In the definition above $\mathbf{n}_{j}$ refers to the vector field normal to $\partial \Omega_{j}$ pointing toward the exterior of $\Omega_{j}$. We will also need a notation to refer to an operator gathering both traces in a single array

$$
\gamma^{j}(u):=\left(\gamma_{\mathrm{D}}^{j}(v), \gamma_{\mathrm{N}}^{j}(v)\right)
$$

Define $\gamma_{\mathrm{D}, c}^{j}, \gamma_{\mathrm{N}, c}^{j}$ in the same manner as $\gamma_{\mathrm{D}}^{j}, \gamma_{\mathrm{N}}^{j}$ with traces taken from the exterior of $\Omega_{j}$, and set in addition $\gamma_{c}^{j}(v):=\left(\gamma_{\mathrm{D}, c}^{j}(v), \gamma_{\mathrm{N}, c}^{j}(v)\right)$. We will also need to refer to mean values and jumps to these trace operators, so we set

$$
\left\{\gamma^{j}(u)\right\}:=\frac{1}{2}\left(\gamma^{j}(u)+\gamma_{c}^{j}(u)\right) \text { and }\left[\gamma^{j}(u)\right]:=\gamma^{j}(u)-\gamma_{c}^{j}(u)
$$

\section{Trace spaces}

We want to recast Problem (1) into variational boundary integral equations. We aim for boundary integral equations set in natural trace spaces. The most fundamental trace space 
we can introduce consists in the multi-trace space [8, Sect. 2.1], the Cartesian product of local traces:

$$
\mathbb{H}(\Sigma):=\mathbb{H}\left(\Gamma_{0}\right) \times \cdots \times \mathbb{H}\left(\Gamma_{n}\right) \quad \text { where } \quad \mathbb{H}\left(\Gamma_{j}\right):=\mathrm{H}^{+\frac{1}{2}}\left(\Gamma_{j}\right) \times \mathrm{H}^{-\frac{1}{2}}\left(\Gamma_{j}\right) .
$$

We endow each $\mathbb{H}\left(\Gamma_{j}\right)$ with the norm $\|(v, q)\|_{\mathbb{H}\left(\Gamma_{j}\right)}:=\left(\|v\|_{\mathrm{H}^{1 / 2}\left(\Gamma_{j}\right)}^{2}+\|q\|_{\mathrm{H}^{-1 / 2}\left(\Gamma_{j}\right)}^{2}\right)^{1 / 2}$, and equip $\mathbb{H}(\Sigma)$ with the norm naturally associated with the cartesian product

$$
\|\mathfrak{u}\|_{\mathbb{H}(\Sigma)}:=\left(\left\|\mathfrak{u}_{0}\right\|_{\mathbb{H}\left(\Gamma_{0}\right)}^{2}+\cdots+\left\|\mathfrak{u}_{n}\right\|_{\mathbb{H}\left(\Gamma_{n}\right)}^{2}\right)^{\frac{1}{2}}
$$

for $\mathfrak{u}=\left(\mathfrak{u}_{0}, \ldots, \mathfrak{u}_{n}\right) \in \mathbb{H}(\Sigma)^{1}$. We write $\langle,\rangle_{\Gamma_{j}}$ for the duality pairing between $\mathrm{H}^{1 / 2}\left(\Gamma_{j}\right)$ and $\mathrm{H}^{-1 / 2}\left(\Gamma_{j}\right)$. In the sequel we shall repeatedly refer to the continuous operator $\gamma$ : $\Pi_{j=0}^{n} \mathrm{H}_{\mathrm{loc}}^{1}\left(\Delta, \bar{\Omega}_{j}\right) \rightarrow \mathbb{H}(\Sigma)$ defined by $\gamma(u):=\left(\gamma_{0}(u), \ldots, \gamma_{n}(u)\right)$, where $\Pi_{j=0}^{n} \mathrm{H}_{\mathrm{loc}}^{1}\left(\Delta, \bar{\Omega}_{j}\right)$ should be understood as the set of $u \in \mathrm{L}_{\text {loc }}^{2}\left(\mathbb{R}^{d}\right)$ such that $\left.u\right|_{\Omega_{j}} \in \mathrm{H}_{\text {loc }}^{1}\left(\Delta, \bar{\Omega}_{j}\right)$ for all $j$. We also need a bilinear duality pairing for $\mathbb{H}\left(\Gamma_{j}\right)$ and $\mathbb{H}(\Sigma)$; we opt for the skew-symmetric version

$$
\llbracket \mathfrak{u}, \mathfrak{v} \rrbracket:=\sum_{j=0}^{n}\left[\mathfrak{u}_{j}, \mathfrak{v}_{j}\right]_{\Gamma_{j}} \quad \text { where } \quad\left[\left(\begin{array}{c}
u_{j} \\
p_{j}
\end{array}\right),\left(\begin{array}{c}
v_{j} \\
q_{j}
\end{array}\right)\right]_{\Gamma_{j}}:=\left\langle u_{j}, q_{j}\right\rangle_{\Gamma_{j}}-\left\langle v_{j}, p_{j}\right\rangle_{\Gamma_{j}} .
$$

This particular choice is well adapted to the forthcoming analysis. Note that under the pairing $\llbracket, \rrbracket$, the space $\mathbb{H}(\Sigma)$ is its own topological dual, and it is easy to show, using the duality between $\mathrm{H}^{1 / 2}\left(\Gamma_{j}\right)$ and $\mathrm{H}^{-1 / 2}\left(\Gamma_{j}\right)$, that the pairing $\llbracket$, 』 induces an isometric isomorphism between $\mathbb{H}(\Sigma)$ and its dual $\mathbb{H}(\Sigma)^{\prime}$, equivalent to the inf-sup condition

$$
\inf _{\mathfrak{v} \in \mathbb{H}(\Sigma)} \sup _{\mathfrak{u} \in \mathbb{H}(\Sigma)} \frac{|\llbracket \mathfrak{u}, \mathfrak{v} \rrbracket|}{\|\mathfrak{u}\|_{\mathbb{H}(\Sigma)}\|\mathfrak{v}\|_{\mathbb{H}(\Sigma)}}=1
$$

Next, as in [8, Sect. 2.2], [10, Sect. 3.1], we introduce the so-called single-trace space that consists in collections of traces that comply with transmission conditions. This space can be defined by

$$
\mathbb{X}(\Sigma):=\cos _{\mathbb{H}(\Sigma)}\left\{\gamma(u)=\left(\gamma^{j}(u)\right)_{j=0}^{n} \mid u \in \mathscr{C}^{\infty}\left(\mathbb{R}^{d}\right)\right\}
$$

where $\operatorname{clos}_{\mathbb{H}(\Sigma)}$ refers to the closure with respect to the norm of $\mathbb{H}(\Sigma)$. By construction, this is a closed subspace of $\mathbb{H}(\Sigma)$. Note also that a function $u \in \mathrm{H}_{\mathrm{loc}}^{1}\left(\Delta, \bar{\Omega}_{0}\right) \times \cdots \times \mathrm{H}_{\mathrm{loc}}^{1}\left(\Delta, \bar{\Omega}_{n}\right)$ satisfies the transmission conditions of (1), if and only if $\gamma(u)=\left(\gamma^{j}(u)\right)_{j=0}^{n} \in \mathbb{X}(\Sigma)$. In particular, if $u \in \mathrm{H}^{1}\left(\Delta, \mathbb{R}^{d}\right)$ then $\gamma(u)=\left(\gamma^{j}(u)\right)_{j=0}^{n} \in \mathbb{X}(\Gamma)$, see $[8, \S 2.2]$ and [7, Lemma 7.4]. In the sequel, we will use this space to enforce transmission conditions. The single-trace space admits a simple weak characterisation, see [8, Prop.2.1].

\section{Lemma 2.1.}

For any $\mathfrak{u} \in \mathbb{H}(\Sigma)$ we have: $\mathfrak{u} \in \mathbb{X}(\Sigma) \Longleftrightarrow \llbracket \mathfrak{u}, \mathfrak{v} \rrbracket=0 \forall \mathfrak{v} \in \mathbb{X}(\Sigma)$.

\footnotetext{
${ }^{1}$ Functions in Dirichlet trace spaces like $\mathrm{H}^{1 / 2}\left(\partial \Omega_{j}\right)$ will be denoted by $u, v, w$, whereas we use $p, q, r$ for Neumann traces. Small fraktur font symbols $\mathfrak{u}, \mathfrak{v}, \mathfrak{w}$ are reserved for Cauchy traces, with an integer subscript indicating restriction to a subdomain boundary.
} 


\section{Summary of potential theory}

In this paragraph, we shall remind the reader of well established results concerning the integral representation of solutions to homogeneous Helmholtz equation in Lipshitz domains. A detailed proof of the statements contained in the present paragraph can be found for example in [25, Chap.3].

Let the function $\mathscr{G}_{\kappa}(\boldsymbol{x})$ refer to the outgoing Green's kernel associated to the Helmholtz operator $-\Delta-\kappa^{2}$. For each subdomain $\Omega_{j}$, for any $(v, q) \in \mathbb{H}\left(\Gamma_{j}\right)$ and any $\boldsymbol{x} \in \mathbb{R}^{d} \backslash \Gamma_{j}$, define the potential operator

$$
\mathrm{G}_{\kappa}^{j}(v, q)(\boldsymbol{x}):=\int_{\Gamma_{j}} q(\boldsymbol{y}) \mathscr{G}_{\kappa}(\boldsymbol{x}-\boldsymbol{y})+v(\boldsymbol{y}) \mathbf{n}_{j}(\boldsymbol{y}) \cdot\left(\nabla \mathscr{G}_{\kappa}\right)(\boldsymbol{x}-\boldsymbol{y}) d \sigma(\boldsymbol{y}) .
$$

The operator $\mathrm{G}_{\kappa}^{j}$ maps continuously $\mathbb{H}\left(\Gamma_{j}\right)$ into $\mathrm{H}^{1}\left(\Delta, \bar{\Omega}_{j}\right) \times \mathrm{H}^{1}\left(\Delta, \mathbb{R}^{d} \backslash \Omega_{j}\right)$, see $[25$, Thm 3.1.16]. In particular $\mathrm{G}_{\kappa}^{j}$ can be applied to a pair of traces of the form $\mathfrak{u}=\gamma^{j}(v)$. This potential operator can be used to write a representation formula for solutions to homogeneous Helmholtz equations, see [25, Thm 3.1.6].

\section{Proposition 3.1.}

Let $u \in \mathrm{H}_{\mathrm{loc}}^{1}\left(\bar{\Omega}_{j}\right)$ satisfy $\Delta u+\kappa_{j}^{2} u=0$ in $\Omega_{j}$. Assume in addition that $u$ is $\kappa_{j}$-outgoing if $j=0$. We have the representation formula

$$
\mathrm{G}_{\kappa_{j}}^{j}\left(\gamma^{j}(u)\right)(\boldsymbol{x})= \begin{cases}u(\boldsymbol{x}) & \text { for } \boldsymbol{x} \in \Omega_{j} \\ 0 & \text { for } \boldsymbol{x} \in \mathbb{R}^{d} \backslash \bar{\Omega}_{j} .\end{cases}
$$

Similarly, if $v \in \mathrm{H}_{\mathrm{loc}}^{1}\left(\mathbb{R}^{d} \backslash \Omega_{j}\right)$ satisfies $\Delta v+\kappa_{j}^{2} v=0$ in $\mathbb{R}^{d} \backslash \bar{\Omega}_{j}$, as well as a radiation condition in the case $j \neq 0$, then we have $\mathrm{G}_{\kappa_{j}}^{j}\left(\gamma_{c}^{j}(v)\right)(\boldsymbol{x})=-v(\boldsymbol{x})$ for $\boldsymbol{x} \in \mathbb{R}^{d} \backslash \bar{\Omega}_{j}$, and $\mathrm{G}_{\kappa_{j}}^{j}\left(\gamma_{c}^{j}(v)\right)(\boldsymbol{x})=0$ for $\boldsymbol{x} \in \Omega_{j}$.

The potential operator (6) also satisfies a remarkable identity, known as jump formula, describing the behaviour of $\mathrm{G}_{\kappa_{j}}^{j}(\mathfrak{v})(\boldsymbol{x})$ as $\boldsymbol{x}$ crosses $\Gamma_{j}=\partial \Omega_{j}$. The notations that we have introduced allow to write it in a very condensed manner, namely

$$
\left[\gamma^{j}\right] \cdot \mathrm{G}_{\kappa_{j}}^{j}(\mathfrak{v})=\mathfrak{v} \quad \forall \mathfrak{v} \in \mathbb{H}\left(\Gamma_{j}\right)
$$

This can also be rewritten $\left[\gamma^{j}\right] \cdot \mathrm{G}_{\kappa_{j}}^{j}=\mathrm{Id}$. We refer the reader to [25, Thm.3.3.1] (the jump formula are more commonly written in the form of four equations in the literature). Proposition 3.1 shows that, if $u$ is solution to a homogeneous Helmholtz equation in $\Omega_{j}$ (and is outgoing if $j=0$ ) then $\gamma^{j} \cdot \mathrm{G}_{\kappa_{j}}^{j}\left(\gamma^{j}(u)\right)=\gamma^{j}(u)$. This actually turns out to be a characterisation of solution to homogeneous Helmholtz equation.

\section{Proposition 3.2.}

Define $\mathcal{C}_{\kappa}^{\text {in }}\left(\Gamma_{j}\right):=\left\{\gamma^{j}(u) \mid u \in \mathrm{H}_{\text {loc }}^{1}\left(\bar{\Omega}_{j}\right), \Delta u+\kappa^{2} u=0\right.$ in $\Omega_{j}, u \kappa$-outgoing if $\left.j=0\right\}$. Then $\gamma^{j} \cdot \mathrm{G}_{\kappa}^{j}: \mathbb{H}\left(\Gamma_{j}\right) \rightarrow \mathbb{H}\left(\Gamma_{j}\right)$ is a continuous projector, called Calderón projector interior to $\Omega_{j}$, whose range coincides with $\mathcal{C}_{\kappa}^{\text {in }}\left(\Gamma_{j}\right)$ i.e. for any $\mathfrak{v} \in \mathbb{H}\left(\Gamma_{j}\right)$

$$
\gamma^{j} \cdot \mathrm{G}_{\kappa}^{j}(\mathfrak{v})=\mathfrak{v} \quad \Longleftrightarrow \quad \mathfrak{v} \in \mathcal{C}_{\kappa}^{\text {in }}\left(\Gamma_{j}\right) \text {. }
$$

Similarly, defining $\mathfrak{C}_{\kappa}^{\text {out }}\left(\Gamma_{j}\right):=\left\{\gamma_{c}^{j}(u) \mid u \in \mathrm{H}_{\mathrm{loc}}^{1}\left(\bar{\Omega}_{j}\right), \Delta u+\kappa^{2} u=0\right.$ in $\mathbb{R}^{d} \backslash \Omega_{j}, u$ outgoing if $\left.j \neq 0\right\}$, we have $\gamma^{j} \cdot \mathrm{G}_{\kappa}^{j}(\mathfrak{v})=0$ if and only if $\mathfrak{v} \in \mathcal{C}_{\kappa}^{\text {out }}\left(\Gamma_{j}\right)$. 
For a detailed proof of this proposition, see [25, Prop.3.6.2]. A direct consequence of this is the so-called Calderón identity $\left(\left\{\gamma^{j}\right\} \cdot \mathrm{G}_{\kappa}^{j}\right)^{2}=\mathrm{Id} / 4$. We shall repeatedly use this characterisation as a convenient way to express wave equations in the subdomains $\Omega_{j}$. Here is another characterisation of the space of Cauchy data which was established in [8, Lemma 6.2].

\section{Lemma 3.1.}

Consider any $j=0, \ldots n$, and any $\kappa \in \mathbb{C} \backslash\{0\}$ such that $\Re e\{\kappa\} \geq 0, \Im m\{\kappa\} \geq 0$. Then for any $\mathfrak{u} \in \mathbb{H}\left(\Gamma_{j}\right)$ we have: $\mathfrak{u} \in \mathcal{C}_{\kappa}^{\text {in }}\left(\Gamma_{j}\right) \Longleftrightarrow[\mathfrak{u}, \mathfrak{v}]_{\Gamma_{j}}=0 \forall \mathfrak{v} \in \mathcal{C}_{\kappa}^{\text {in }}\left(\Gamma_{j}\right)$. Similarly we have $\mathfrak{u} \in \mathcal{C}_{\kappa}^{\text {out }}\left(\Gamma_{j}\right) \Longleftrightarrow[\mathfrak{u}, \mathfrak{v}]_{\Gamma_{j}}=0 \forall \mathfrak{v} \in \mathcal{C}_{\kappa}^{\text {out }}\left(\Gamma_{j}\right)$.

The results that we have stated above hold for any $j=0 \ldots n$. They can be re-expressed in a matrix form, which will help reducing notations. First we introduce the continuous operator $\mathrm{A}: \mathbb{H}(\Sigma) \rightarrow \mathbb{H}(\Sigma)$ defined by

$$
\frac{1}{2} \llbracket A \mathfrak{u}, \mathfrak{v} \rrbracket:=\sum_{j=0}^{n}\left[\left\{\gamma^{j}\right\} \cdot \mathrm{G}_{\kappa_{j}}^{j}\left(\mathfrak{u}_{j}\right), \mathfrak{v}_{j}\right]_{\Gamma_{j}} .
$$

for all $\mathfrak{u}=\left(\mathfrak{u}_{j}\right)_{j=0}^{n}, \mathfrak{v}=\left(\mathfrak{v}_{j}\right)_{j=0}^{n} \in \mathbb{H}(\Sigma)$. We also set $\mathcal{C}^{\alpha}(\Sigma):=\mathcal{C}_{\kappa_{0}}^{\alpha}\left(\Gamma_{0}\right) \times \cdots \times \mathcal{C}_{\kappa_{n}}^{\alpha}\left(\Gamma_{n}\right)$ for $\alpha=$ in, out. Observe that $(\mathrm{Id} \pm \mathrm{A}) / 2$ are projectors, according to Proposition 3.2, with $\operatorname{Ker}(\mathrm{A}-\mathrm{Id})=\operatorname{Range}(\mathrm{A}+\mathrm{Id})=\mathrm{C}^{\mathrm{in}}(\Sigma)$ and $\operatorname{Ker}(\mathrm{A}+\mathrm{Id})=\operatorname{Range}(\mathrm{A}-\mathrm{Id})=\operatorname{e}^{\text {out }}(\Sigma)$. The notations we have just introduced lead to the following compact reformulation of the well-posedness of (1), see [8, Prop.6.1] for a detailed proof.

\section{Lemma 3.2.}

$\mathbb{X}(\Sigma) \oplus \operatorname{e}^{\text {in }}(\Sigma)=\mathbb{H}(\Sigma)$.

\section{Local multi-trace formulation}

Now we would like to recall the derivation of the local multi-trace formulation, that was first introduced in [17]. In the present section we will only recall already established results concerning local multi-trace formulation, not giving a complete overview, and refer the reader to [17] for detailed proof of these results.

A key ingredient of the local multi-trace theory is an operator yielding a characterisation of transmission conditions of (1). Let us denote $\mathbb{L}^{2}(\Sigma):=\Pi_{j=0}^{n}\left(\mathrm{~L}^{2}\left(\Gamma_{j}\right) \times \mathrm{L}^{2}\left(\Gamma_{j}\right)\right)$. For $\mathfrak{u}=$ $\left(u_{j}, p_{j}\right)_{j=0}^{n} \in \mathbb{L}^{2}(\Sigma)$, and $\mathfrak{v}=\left(v_{j}, q_{j}\right)_{j=0}^{n} \in \mathbb{L}^{2}(\Sigma)$, we define the transmission operator $\Pi$ : $\mathbb{L}^{2}(\Sigma) \rightarrow \mathbb{L}^{2}(\Sigma)$ by

$$
\mathfrak{v}=\Pi(\mathfrak{u}) \quad \Longleftrightarrow \quad\left\{\begin{array}{l}
v_{j}=+u_{k} \\
q_{j}=-p_{k}
\end{array} \quad \text { on } \Gamma_{j, k} \quad \forall j, k=0 \ldots n .\right.
$$

Clearly, for any function $u \in \mathrm{H}_{\mathrm{loc}}^{2}\left(\mathbb{R}^{d}\right)$ we have $\gamma(u):=\left(\gamma^{j}(u)\right)_{j=0}^{n} \in \mathbb{L}^{2}(\Sigma)$ and $\gamma(u)=$ $\Pi(\gamma(u))$. Conversely, considering any function $u \in \mathrm{L}_{\text {loc }}^{2}\left(\mathbb{R}^{d}\right)$ such that $\left.u\right|_{\Omega_{j}} \in \mathrm{H}_{\mathrm{loc}}^{2}\left(\bar{\Omega}_{j}\right)$ for all $j=0 \ldots n$, then $\gamma(u) \in \mathbb{L}^{2}(\Sigma)$ is well defined, and if $\gamma(u)=\Pi(\gamma(u))$ then we have $u \in \mathrm{H}_{\mathrm{loc}}^{2}\left(\mathbb{R}^{d}\right)$. Routine calculus shows that the transmission operator satisfies the following remarkable identities

$$
\Pi^{2}=\operatorname{Id}, \quad \overline{\Pi(\mathfrak{v})}=\Pi(\overline{\mathfrak{v}}) \quad \text { and } \quad \llbracket \Pi(\mathfrak{u}), \mathfrak{v} \rrbracket=\llbracket \Pi(\mathfrak{v}), \mathfrak{u} \rrbracket \quad \forall \mathfrak{u}, \mathfrak{v} \in \mathbb{L}^{2}(\Sigma) .
$$


As is readily checked, the operator $\Pi$ maps continuously $\mathbb{H}(\Sigma)$ onto $\mathbb{H}(\Sigma)$ under the assumption that $\Gamma_{j, k}$ is a Lipschitz manifold without boundary for any $j, k$, which corresponds to situations where there is no junction point i.e. points where at least three subdomains abut. Without this assumption, $\Pi$ does not map $\mathbb{H}(\Sigma)$ into itself. We actually have the continuous mapping $\Pi: \mathbb{H}(\Sigma) \rightarrow \widetilde{\mathbb{H}}(\Sigma)^{\prime}$ where $\widetilde{\mathbb{H}}(\Sigma)^{\prime}$ is the topological dual to the space

$$
\begin{aligned}
\widetilde{\mathbb{H}}(\Sigma):=\left\{\mathfrak{v}=\left(v_{j}, q_{j}\right)_{j=0}^{n} \in \mathbb{H}(\Sigma) \mid\right. & \left.v_{j}\right|_{\Gamma_{j, k}} \in \widetilde{\mathrm{H}}^{+\frac{1}{2}}\left(\Gamma_{j, k}\right), \\
\left.q_{j}\right|_{\Gamma_{j, k}} & \left.\in \widetilde{\mathrm{H}}^{-\frac{1}{2}}\left(\Gamma_{j, k}\right) \quad \forall j, k=0 \ldots n\right\}
\end{aligned}
$$

equipped with

$$
\|\mathfrak{v}\|_{\widetilde{\mathbb{H}}(\Sigma)}:=\left(\sum_{j=0}^{n} \sum_{\substack{k=0 \\ k \neq j}}^{n}\left\|v_{j}\right\|_{\widetilde{\mathrm{H}}^{1 / 2}\left(\Gamma_{j, k}\right)}^{2}+\left\|q_{j}\right\|_{\widetilde{\mathrm{H}}^{-1 / 2}\left(\Gamma_{j, k}\right)}^{2}\right)^{1 / 2}
$$

where, following [19, Chap.3], for $\sigma= \pm 1 / 2$ we adopt the standard definitions $\mathrm{H}^{\sigma}\left(\Gamma_{j, k}\right)=$ $\left\{\left.\varphi\right|_{\Gamma_{j, k}} \varphi \in \mathrm{H}^{\sigma}\left(\Gamma_{j}\right)\right\}$ and $\widetilde{\mathrm{H}}^{\sigma}\left(\Gamma_{j, k}\right):=\mathrm{H}^{-\sigma}\left(\Gamma_{j, k}\right)^{\prime}$.

The space $\widetilde{\mathbb{H}}(\Sigma)$ equipped with the norm above is a Banach space. We have $\widetilde{\mathbb{H}}(\Sigma) \subset$ $\mathbb{H}(\Sigma)=\mathbb{H}(\Sigma)^{\prime} \subset \widetilde{\mathbb{H}}(\Sigma)^{\prime}$. Besides, $\widetilde{\mathbb{H}}(\Sigma)$ is dense in $\mathbb{H}(\Sigma)$ in the sense of \|\|$_{\mathbb{H}(\Sigma)}$, and the norm \|\|$_{\widetilde{H}(\Sigma)}$ is strictly stronger than the norm \|\|$_{\mathbb{H}(\Sigma)}$, see $[17, \S 2.2 .2]$. Elementary arguments on trace spaces show that, for any $\mathfrak{u} \in \mathbb{H}(\Sigma)$, we have $\mathfrak{u} \in \mathbb{X}(\Sigma) \Longleftrightarrow \mathfrak{u}=\Pi(\mathfrak{u})$. Since $\Pi^{2}=\mathrm{Id}$, this can be simply rewritten in the following manner.

\section{Lemma 4.1.}

range $(\Pi+\mathrm{Id})=\mathbb{X}(\Sigma)$.

Now consider $\mathfrak{u}=\left(\gamma^{j}(u)\right)_{j=0}^{n}$ the traces of the unique solution $u$ to Problem (1), and denote $\mathfrak{u}_{\text {inc }}:=\left(\gamma^{0}\left(u_{\text {inc }}\right), 0, \ldots, 0\right)$. The homogeneous wave equation satisfied by $u$ in each subdomain can be reformulated by means of Calderón projectors $(A-I d)\left(\mathfrak{u}-\mathfrak{u}_{\text {inc }}\right)=0$. Moreover we must have $\mathfrak{u}=\Pi(\mathfrak{u})$ since $u$ satisfies the transmission conditions of (1). Setting $\mathfrak{f}:=(\mathrm{A}-\mathrm{Id}) \mathfrak{u}_{\mathrm{inc}}$, this implies

$$
\left\{\begin{array}{l}
\mathfrak{u} \in \mathbb{H}(\Sigma) \quad \text { and } \\
\llbracket(\mathrm{A}-\Pi) \mathfrak{u}, \mathfrak{v} \rrbracket=\llbracket \mathfrak{f}, \mathfrak{v} \rrbracket \quad \forall \mathfrak{v} \in \widetilde{\mathbb{H}}(\Sigma) .
\end{array}\right.
$$

For this variational formulation, there is existence [17, Thm.11] and uniqueness [17, Thm.9] to its solution.

\section{Lemma 4.2.}

For any $\mathfrak{f} \in \mathbb{H}(\Sigma)$, the formulation (10) admits a unique solution $\mathfrak{u} \in \mathbb{H}(\Sigma)$ that depends continuously on $\mathfrak{f}$. In particular $\operatorname{Ker}(\mathrm{A}-\Pi)=\{0\}$.

Whether this formulation satisfies a Garding inequality remains an open question though. For this reason, so far, uniform discrete inf-sup conditions (and hence quasi-uniform approximation property) for Galerkin discretisations of (10) could only be established in the particular case of 2-D scalar problems. 


\section{Reformulation of transmission conditions}

The difficulties arising in the analysis of (10) are mainly due to the presence of junction points. Indeed, with such points, the operator $\Pi$ is no more continuous in $\mathbb{H}(\Sigma)$ that, at the same time, appears as a natural functional setting for the boundary integral formulations of the transmission problem (1). In the present paper, we propose a specific additional treatment of junction points that leads to a regularised operator $\widetilde{\Pi}$ for which continuity in the norm of $\mathbb{H}(\Sigma)$ is restored.

\subsection{Treatment of junction points}

The particular treatment that we propose will rely on integral operators. In this paragraph, $\mathscr{K}_{0}$ will refer to a Green kernel of an Helmholtz equation i.e. we will assume that it satisfies $-\Delta \mathscr{K}_{0}-\lambda^{2} \mathscr{K}_{0}=\delta_{0}$ in $\mathbb{R}^{d}$ in the sense of distributions, for some $\lambda \in \mathbb{C}$. The choice of $\lambda$ can be decorrelated from the wave numbers $\kappa_{j}$ coming into play in (1). So the Green kernels $\mathscr{K}_{0}$ and $\mathscr{G}$ are independent.

We also consider a function $\mathscr{K} \in \mathscr{C}^{\infty}\left(\mathbb{R}^{d} \backslash\{0\}\right)$ such that $\mathscr{K}$ coincides with $\mathscr{K}_{0}$ in a neighbourhood of 0 . It is important to note that, the kernel $\mathscr{K}(\boldsymbol{x})$ shares the same singularity at $\boldsymbol{x}=0$ as $\mathscr{K}_{0}(\boldsymbol{x})$. Except in a neighbourhood of 0 , we do not impose that $\mathscr{K}(\boldsymbol{x})$ satisfies any particular equation. It does not a priori need to be the Green kernel of any equation. In addition, the $\mathscr{K}$ may be chosen so as to admit a bounded support located closely around $\boldsymbol{x}=0$. For any $(v, q) \in \mathbb{H}\left(\Gamma_{j}\right)$, set

$$
\mathrm{K}^{j}(v, q)(\boldsymbol{x}):=\int_{\partial \Omega_{j}} \mathscr{K}(\boldsymbol{x}-\boldsymbol{y}) q(\boldsymbol{y})+\mathbf{n}_{j}(\boldsymbol{y}) \cdot(\nabla \mathscr{K})(\boldsymbol{x}-\boldsymbol{y}) v(\boldsymbol{y}) d \sigma(\boldsymbol{y})
$$

Because the kernel $\mathscr{K}$ only differs from a Green's kernel up to a smooth factor, the operators $\mathrm{K}^{j}$ satisfy many remarkable properties inherited from standard potential operators. For example, they satisfy a symmetry property, and jump formula.

\section{Lemma 5.1.}

The operator $\mathrm{K}^{j}$ maps continuously $\mathbb{H}\left(\Gamma_{j}\right)$ into $\mathrm{H}^{1}\left(\Delta, \Omega_{j}\right) \times \mathrm{H}_{\text {loc }}^{1}\left(\Delta, \mathbb{R}^{d} \backslash \Omega_{j}\right)$. Moreover, for any $\mathfrak{u}, \mathfrak{v} \in \mathbb{H}\left(\Gamma_{j}\right), \mathfrak{w} \in \mathbb{H}\left(\Gamma_{k}\right)$ with $k \neq j$, we have the following elementary properties:

$$
\begin{aligned}
& \text { i) }\left[\gamma^{j}\right] \cdot \mathrm{K}^{j}(\mathfrak{u})=\mathfrak{u}, \\
& \text { ii) }\left[\left\{\gamma^{j}\right\} \cdot \mathrm{K}^{j}(\mathfrak{u}), \mathfrak{v}\right]_{\Gamma_{j}}=\left[\left\{\gamma^{j}\right\} \cdot \mathrm{K}^{j}(\mathfrak{v}), \mathfrak{u}\right]_{\Gamma_{j}}, \\
& \text { iii) }\left[\gamma^{j} \cdot \mathrm{K}^{k}(\mathfrak{w}), \mathfrak{u}\right]_{\Gamma_{j}}=\left[\gamma^{k} \cdot \mathrm{K}^{j}(\mathfrak{u}), \mathfrak{w}\right]_{\Gamma_{k}} .
\end{aligned}
$$

\section{Proof:}

Let $\mathrm{K}_{0}^{j}$ refer to the same operator as $\mathrm{K}^{j}$ but constructed with the Green kernel $\mathscr{K}_{0}$ instead of $\mathscr{K}$, and set $\mathrm{K}_{*}^{j}:=\mathrm{K}^{j}-\mathrm{K}_{0}^{j}$. The properties announced above already hold with $\mathrm{K}_{0}^{j}$ instead of $\mathrm{K}^{j}$, see for example [13]. Besides the kernel of $\mathrm{K}_{*}^{j}$ is $\mathscr{C}^{\infty}$ since $\mathscr{K}$ and $\mathscr{K}_{0}$ coincide in a neighbourhood of 0 . This proves the continuity properties announced for $\mathrm{K}^{j}$, and shows in addition that $\left[\gamma^{j}\right] \cdot K_{*}^{j}=0$. Moreover, it is a straightforward consequence of Fubini's theorem, that ii) and iii) are verified with $\mathrm{K}^{j}$ replaced by $\mathrm{K}_{*}^{j}$. Since we also know that ii) and iii) are verified with $\mathrm{K}_{0}^{j}$ (see lemmas 3.6 and 3.7 in [9] for example), this concludes the proof. 
We may rely on combinations of all operators $\mathrm{K}^{j}$ for reformulating transmission conditions across interfaces. Indeed consider the sum of the $\mathrm{K}^{j}$ over all subdomains i.e. for any $\mathfrak{u}=$ $\left(\mathfrak{u}_{j}\right)_{j=0}^{n} \in \mathbb{H}(\Sigma)$, set

$$
\mathrm{K}(\mathfrak{u})(\boldsymbol{x}):=\sum_{j=0}^{n} \mathrm{~K}^{j}\left(\mathfrak{u}_{j}\right)(\boldsymbol{x}), \quad \text { for } \quad \boldsymbol{x} \in \mathbb{R}^{d} \backslash \Sigma .
$$

This operator is close to the multi-potential operator introduced in $[6,11]$. It maps continuously $\mathbb{H}(\Sigma)$ into $\Pi_{j=0}^{n} \mathrm{H}_{\text {loc }}^{1}\left(\Delta, \bar{\Omega}_{j}\right)$. It is important to note, though, that the same kernel has been considered for all terms in the sum (12).

Recall that we have introduced a global trace operator defined by $\gamma(v):=\left(\gamma^{0}(v), \ldots, \gamma^{n}(v)\right) \in$ $\mathbb{H}(\Sigma)$. We can combine this global trace with the operator (12) which yields a continuous map $\gamma \cdot \mathrm{K}: \mathbb{H}(\Sigma) \rightarrow \mathbb{H}(\Sigma)$. This operator satisfies several remarkable properties.

\section{Lemma 5.2.}

$\llbracket \gamma \cdot \mathrm{K}(\mathfrak{u}), \mathfrak{v} \rrbracket=\llbracket \gamma \cdot \mathrm{K}(\mathfrak{v}), \mathfrak{u} \rrbracket+\llbracket \mathfrak{u}, \mathfrak{v} \rrbracket$

This result is obtained straightforwardly by expanding K according to (12), and then applying the properties stated in Lemma 5.1, together with the simple identity $\gamma^{j}=\left\{\gamma^{j}\right\}+\frac{1}{2}\left[\gamma^{j}\right]$.

\section{Lemma 5.3.}

$\mathrm{K}(\mathfrak{u})(\boldsymbol{x})=0 \quad \forall \mathfrak{u} \in \mathbb{X}(\Sigma), \forall \boldsymbol{x} \in \mathbb{R}^{d}$.

\section{Proof:}

Choose an arbitrary $\boldsymbol{x} \in \mathbb{R}^{d} \backslash \Sigma$, and let $\chi \in \mathscr{C}^{\infty}\left(\mathbb{R}^{d}\right)$ refer to a cut-off function such that $\chi(\boldsymbol{y})=1$ for $\boldsymbol{y}$ in a neighbourhood of $\Sigma, \chi(\boldsymbol{y})=0$ for $\boldsymbol{y}$ in a neighbourhood of $\boldsymbol{x}$ and $\operatorname{supp}(\chi)$ is bounded. Denote $\mathscr{K}^{\boldsymbol{x}}(\boldsymbol{y}):=\chi(\boldsymbol{y}) \mathscr{K}(\boldsymbol{x}-\boldsymbol{y})$ so that $\mathscr{K}^{\boldsymbol{x}} \in \mathscr{C}^{\infty}\left(\mathbb{R}^{d}\right)$ and $\mathscr{K}^{\boldsymbol{x}}$ has compact support. Take an arbitrary $u \in \mathscr{C}^{\infty}\left(\mathbb{R}^{d}\right)$. Using the explicit definitions (11) and (12) we obtain

$$
\begin{aligned}
\mathrm{K}(\gamma(u))(\boldsymbol{x}) & =\sum_{j=0}^{n} \int_{\partial \Omega_{j}} \gamma_{\mathrm{D}}^{j}\left(\mathscr{K}^{\boldsymbol{x}}\right) \gamma_{\mathrm{N}}^{j}(u)-\gamma_{\mathrm{N}}^{j}\left(\mathscr{K}^{\boldsymbol{x}}\right) \gamma_{\mathrm{D}}^{j}(u) d \sigma \\
& =\sum_{j=0}^{n} \int_{\Omega_{j}} \mathscr{K}^{\boldsymbol{x}} \Delta u-u \Delta \mathscr{K}^{\boldsymbol{x}} d \boldsymbol{y}=\int_{\mathbb{R}^{d}} \mathscr{K}^{\boldsymbol{x}} \Delta u-u \Delta \mathscr{K}^{\boldsymbol{x}} d \boldsymbol{y}=0 .
\end{aligned}
$$

Since this holds for any $\boldsymbol{x} \in \mathbb{R}^{d} \backslash \Sigma$, we conclude that $\mathrm{K}(\gamma(u))$ for any $u \in \mathscr{C}^{\infty}\left(\mathbb{R}^{d}\right)$. In addition, since the elements of the form $\gamma(u)$ for $u \in \mathscr{C}^{\infty}\left(\mathbb{R}^{d}\right)$ are dense in $\mathbb{X}(\Sigma)$, according to (5), this concludes the proof.

\section{Lemma 5.4.}

$\mathfrak{u}-\gamma \cdot \mathrm{K}(\mathfrak{u}) \in \mathbb{X}(\Sigma) \quad \forall \mathfrak{u} \in \mathbb{H}(\Sigma)$.

\section{Proof:}

Take $\mathfrak{u} \in \mathbb{H}(\Sigma)$. According to Lemma 5.2 and Lemma 5.3, for any $\mathfrak{v} \in \mathbb{X}(\Sigma)$ we have $\llbracket \mathfrak{u}-\gamma \cdot \mathrm{K}(\mathfrak{u}), \mathfrak{v} \rrbracket=\llbracket \mathfrak{u}, \mathfrak{v} \rrbracket-\llbracket \gamma \cdot \mathrm{K}(\mathfrak{u}), \mathfrak{v} \rrbracket=-\llbracket \gamma \cdot \mathrm{K}(\mathfrak{v}), \mathfrak{u} \rrbracket=0$. Now there only remains to apply Lemma 2.1 which characterises the elements of $\mathbb{X}(\Sigma)$. 


\section{Corollary 5.1.}

The operator $\gamma \cdot \mathrm{K}: \mathbb{H}(\Sigma) \rightarrow \mathbb{H}(\Sigma)$ is a continuous projector with $\operatorname{Ker}(\gamma \cdot \mathrm{K})=\mathbb{X}(\Sigma)$.

\section{Proof:}

Lemma 5.3 clearly shows that $\mathbb{X}(\Sigma) \subset \operatorname{Ker}(\gamma \cdot \mathrm{K})$. On the other hand, take any $\mathfrak{u} \in$ $\operatorname{Ker}(\gamma \cdot \mathrm{K})$. Applying Lemma 5.4, we obtain directly $\mathfrak{u}=\mathfrak{u}-\gamma \cdot \mathrm{K}(\mathfrak{u}) \in \mathbb{X}(\Sigma)$. We conclude that $\operatorname{Ker}(\gamma \cdot \mathrm{K})=\mathbb{X}(\Sigma)$. This, combined with Lemma 5.4, proves that $\gamma \cdot \mathrm{K} \cdot(\mathrm{Id}-\gamma \cdot \mathrm{K})=0$. We deduce that $(\gamma \cdot \mathrm{K})^{2}=\gamma \cdot \mathrm{K} \cdot(\gamma \cdot \mathrm{K}-\mathrm{Id})+\gamma \cdot \mathrm{K}=\gamma \cdot \mathrm{K}$.

This last result shows that the operator $\gamma \cdot \mathrm{K}$ can be used to characterise the elements of $\mathbb{X}(\Sigma)$, and to propose alternatives to operator $\Pi$. The following proposition yields definition to possible alternative operators. The proof is a direct consequence of Lemma 5.2 to 5.4 and Corollary 5.1.

\section{Proposition 5.1.}

Consider the operator $\mathrm{K}$ given by (12), and define the continuous operator $\mathrm{Q}:=\mathrm{Id}-2 \gamma \cdot \mathrm{K}$. We have $\mathrm{Q}^{2}=\operatorname{Id}$ and $\llbracket \mathrm{Q}(\mathfrak{u}), \mathfrak{v} \rrbracket=\llbracket \mathrm{Q}(\mathfrak{v}), \mathfrak{u} \rrbracket$ for all $\mathfrak{u}, \mathfrak{v} \in \mathbb{H}(\Sigma)$. Moreover for any $\mathfrak{u} \in \mathbb{H}(\Sigma)$, we have $\mathfrak{u} \in \mathbb{X}(\Sigma) \Longleftrightarrow \mathfrak{u}=\mathrm{Q}(\mathfrak{u})$.

Clearly the operator $\mathrm{Q}$ introduced above is an isomorphism since $\mathrm{Q}^{2}=\mathrm{Id}$. It actually satisfies in addition a generalised Garding inequality.

\section{Proposition 5.2.}

Define $\Theta: \mathbb{H}(\Sigma) \rightarrow \mathbb{H}(\Sigma)$ by $\Theta(\mathfrak{u})=\left(-u_{j}, p_{j}\right)_{j=0}^{n}$ for any $\mathfrak{u}=\left(u_{j}, p_{j}\right) \in \mathbb{H}(\Sigma)$. Then there exists a compact operator $\mathrm{R}: \mathbb{H}(\Sigma) \rightarrow \mathbb{H}(\Sigma)$ and a constant $C>0$ such that

$$
\Re e\{\llbracket(-\mathrm{Q}+\mathrm{R}) \mathfrak{u}, \Theta(\overline{\mathfrak{u}}) \rrbracket\} \geq C\|\mathfrak{u}\|_{\mathbb{H}(\Sigma)}^{2} \quad \forall \mathfrak{u} \in \mathbb{H}(\Sigma) .
$$

\section{Proof:}

Let $\mathscr{K}_{\bullet}$ refer to the unique distributional solution of $-\Delta \mathscr{K}_{\bullet}+\mathscr{K}_{\bullet}=\delta_{0}$ in $\mathbb{R}^{d}$ and $\lim _{|\boldsymbol{x}| \rightarrow \infty} \mathscr{K}_{\bullet}(\boldsymbol{x})=0$. Denote K• and Q• the operator defined by (11)-(12) and Proposition 5.1 constructed with this kernel. The operator $\mathrm{K}-\mathrm{K}$ • is compact, as it involves smoother kernels of the form $\mathscr{K}(\boldsymbol{x})-\mathscr{K}_{\bullet}(\boldsymbol{x})$, see [25, Lemma 3.9.8] for example. This implies that Q- Q• is compact. Finally, since the operator Q• satisfies a Garding inequality such as (13) according to [8, Prop.10.3], this concludes the proof.

\subsection{Quasi-local transmission operator}

In the case where the skeleton $\Sigma$ does not involve any junction point (i.e. points where three or more subdomains abut), the local multi-trace operator $\mathrm{A}-\Pi$ maps $\mathbb{H}(\Sigma)$ onto itself, and satisfies a Garding inequality. Hence, the main motivation for considering Q, is the treatment of junction points. A fortiori, for writing transmission conditions, it would seem desirable to use Q only at junction points, and use the operator $\Pi$ anywhere else on $\Sigma$. In this section, we present the construction of an operator $\widetilde{\Pi}$ similar to $\Pi$, but involving the operator $Q$ in fixed arbitrarily small neighbourhoods of junction points. Define

$$
\mathscr{J}=\bigcup_{0 \leq i<j<k \leq n} \partial \Omega_{i} \cap \partial \Omega_{j} \cap \partial \Omega_{k} .
$$

By definition, these are the points adjacent to at least three subdomains. For $\mathbb{R}^{d}=\mathbb{R}^{2}$ it consists in a finite set of points, and for $\mathbb{R}^{d}=\mathbb{R}^{3}$ it consists in a finite union of Lipschitz 
curves. For $\epsilon>0$ define $\mathscr{V}_{\epsilon}:=\left\{\boldsymbol{x} \in \mathbb{R}^{d} \mid \operatorname{dist}(\boldsymbol{x}, \mathscr{J})<\epsilon\right\}$. We suppose that $\epsilon$ is chosen small enough to guarantee that

$$
\Gamma_{j, k} \backslash \mathscr{N}_{2 \epsilon} \neq \emptyset \quad \forall j, k=0 \ldots n .
$$

We consider a $\mathscr{C}^{\infty}$ cut-off function $\psi: \mathbb{R}^{d} \rightarrow \mathbb{R}$ such that $\psi(\boldsymbol{x})=1$ for $\boldsymbol{x} \in \mathscr{V}_{\epsilon}$ and $\psi(\boldsymbol{x})=0$ for $\boldsymbol{x} \in \mathbb{R}^{d} \backslash \mathscr{V}_{2 \epsilon}$, and define $\chi:=1-\psi^{2}$. In particular $\chi$ vanishes over a neighbourhood of junctions points. In a two dimensional geometrical context, the situation looks as depicted in Figure 1 below.

In the sequel, for any $\mathfrak{u}=\left(u_{j}, p_{j}\right)_{j=0}^{n} \in \mathbb{H}(\Sigma)$, we will denote $\chi \mathfrak{u}:=\left(\chi u_{j}, \chi p_{j}\right)_{j=0}^{n}$. And we adopt a similar notation with $\psi$. This implies that $\llbracket \chi \mathfrak{u}, \mathfrak{v} \rrbracket=\llbracket \mathfrak{u}, \chi \mathfrak{v} \rrbracket$, and also $\llbracket \mathfrak{u}, \mathfrak{v} \rrbracket=$ $\llbracket \chi \mathfrak{u}, \mathfrak{v} \rrbracket+\llbracket \psi \mathfrak{u}, \psi \mathfrak{v} \rrbracket$. Next we define the continuous operator $\widetilde{\Pi}: \mathbb{H}(\Sigma) \rightarrow \mathbb{H}(\Sigma)$ by

$$
\llbracket \widetilde{\Pi}(\mathfrak{u}), \mathfrak{v} \rrbracket:=\llbracket \Pi(\chi \mathfrak{u}), \mathfrak{v} \rrbracket+\llbracket Q(\psi \mathfrak{u}), \psi \mathfrak{v} \rrbracket \quad \forall \mathfrak{u}, \mathfrak{v} \in \mathbb{H}(\Sigma) .
$$

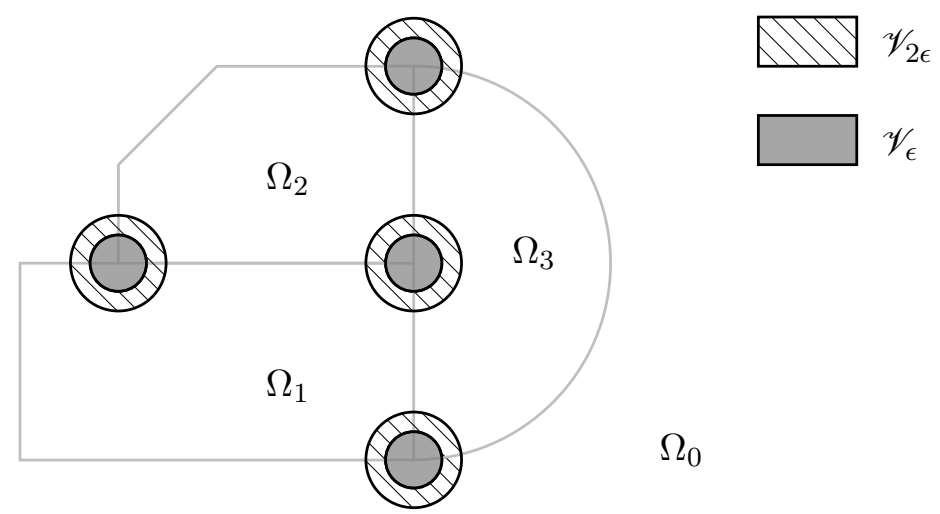

Figure 1: Support of cut-off functions localized at junction points in a typical 2-D multi-subdomain geometry.

Clearly $\widetilde{\Pi}: \mathbb{H}(\Sigma) \rightarrow \mathbb{H}(\Sigma)$ is continuous since $\chi$ vanishes at junction points. It coincides with $\Pi$ except in $\mathscr{V} / 2 \epsilon$, where it admits a non-local expression given by the operator Q. This is the reason why $\widetilde{\Pi}$ may be considered "quasi-local". The operator $\widetilde{\Pi}$ is constructed as an interpolation between $\Pi$ and Q. As such, it shares several properties with these operators.

\section{Proposition 5.3.}

We have $\mathfrak{u}+\widetilde{\Pi}(\mathfrak{u}) \in \mathbb{X}(\Sigma)$ for all $\mathfrak{u} \in \mathbb{H}(\Sigma)$, and $\operatorname{Ker}(\operatorname{Id}-\widetilde{\Pi})=\mathbb{X}(\Sigma)$.

\section{Proof:}

To prove the first property pick any $\mathfrak{u} \in \mathbb{H}(\Sigma)$, and take an arbitrary $\mathfrak{v} \in \mathbb{X}(\Sigma)$. From Lemma 2.1 and Lemma 4.1 we conclude that $\llbracket \chi \mathfrak{u}+\Pi(\chi \mathfrak{u}), \mathfrak{v} \rrbracket=0$. Note in addition that $\psi \mathfrak{v} \in \mathbb{X}(\Sigma)$. As a consequence, since $(-\mathrm{Id}+\mathrm{Q}) \mathfrak{w}=0 \forall \mathfrak{w} \in \mathbb{X}(\Sigma)$ according to Proposition 5.1 , we obtain

$$
\begin{aligned}
\llbracket \mathfrak{u}+\widetilde{\Pi}(\mathfrak{u}), \mathfrak{v} \rrbracket & =\llbracket \chi \mathfrak{u}+\Pi(\chi \mathfrak{u}), \mathfrak{v} \rrbracket+\llbracket \psi \mathfrak{u}+\mathrm{Q}(\psi \mathfrak{u}), \psi \mathfrak{v} \rrbracket \\
& =\llbracket-\psi \mathfrak{v}+\mathrm{Q}(\psi \mathfrak{v}), \psi \mathfrak{u} \rrbracket=0
\end{aligned}
$$

Since $\mathfrak{v} \in \mathbb{X}(\Sigma)$ was chosen arbitrarily, we conclude from Lemma 2.1 that $\mathfrak{u}+\widetilde{\Pi}(\mathfrak{u}) \in \mathbb{X}(\Sigma)$. As this holds for any $\mathfrak{u} \in \mathbb{H}(\Sigma)$, this proves the first statement. 
Now let us prove the second property of the proposition. Pick any $\mathfrak{u} \in \mathbb{X}(\Sigma)$, and take an arbitrary $\mathfrak{v} \in \mathbb{H}(\Sigma)$. Note that $\chi \mathfrak{u} \in \mathbb{X}(\Sigma)$ and $\chi \mathfrak{u}$ vanishes in the neighbourhood of junction points, so that $\Pi(\chi \mathfrak{u})=\chi \mathfrak{u}$ which implies $\llbracket \chi \mathfrak{u}-\Pi(\chi \mathfrak{u}), \mathfrak{v} \rrbracket=0$. Similarly we have $(-\mathrm{Id}+\mathrm{Q})(\psi \mathfrak{u})=0$ since $\psi \mathfrak{u} \in \mathbb{X}(\Sigma)$, so $\llbracket \psi \mathfrak{u}-\mathrm{Q}(\psi \mathfrak{u}), \psi \mathfrak{v} \rrbracket=0$. The definition (14) of $\widetilde{\Pi}$ then yields

$$
\llbracket \mathfrak{u}-\widetilde{\Pi}(\mathfrak{u}), \mathfrak{v} \rrbracket=\llbracket \chi \mathfrak{u}-\Pi(\chi \mathfrak{u}), \mathfrak{v} \rrbracket+\llbracket \psi \mathfrak{u}-\mathrm{Q}(\psi \mathfrak{u}), \psi \mathfrak{v} \rrbracket=0 \quad \forall \mathfrak{v} \in \mathbb{H}(\Sigma) .
$$

This shows that $\mathbb{X}(\Sigma) \subset \operatorname{Ker}(\operatorname{Id}-\widetilde{\Pi})$. Now let us take $\mathfrak{u} \in \operatorname{Ker}(\operatorname{Id}-\widetilde{\Pi})$ which can be written $2 \mathfrak{u}=\mathfrak{u}+\widetilde{\Pi}(\mathfrak{u})$. The first part of the proof then shows that $\mathfrak{u} \in \mathbb{X}(\Sigma)$.

\section{Proposition 5.4.}

We have $(\widetilde{\Pi})^{2}=\mathrm{Id}$, and $\llbracket \widetilde{\Pi}(\mathfrak{u}), \mathfrak{v} \rrbracket=\llbracket \widetilde{\Pi}(\mathfrak{v}), \mathfrak{u} \rrbracket \forall \mathfrak{u}, \mathfrak{v} \in \mathbb{H}(\Sigma)$. Moreover for any $\mathfrak{u} \in \mathbb{H}(\Sigma)$, we have $\mathfrak{u} \in \mathbb{X}(\Sigma) \Longleftrightarrow \mathfrak{u}=\widetilde{\Pi}(\mathfrak{u})$.

\section{Proof:}

It is a direct consequence of the previous proposition that $(\mathrm{Id}-\widetilde{\Pi})(\mathrm{Id}+\widetilde{\Pi})=0=\mathrm{Id}-(\widetilde{\Pi})^{2}$. To prove the second property, observe directly from the definition (8) of $\Pi$ that $\chi \Pi=\Pi \chi$. Moreover we know from (9) and Proposition 5.1 that $\llbracket \Pi(\mathfrak{u}), \mathfrak{v} \rrbracket=\llbracket \Pi(\mathfrak{v}), \mathfrak{u} \rrbracket$ and $\llbracket Q(\mathfrak{u}), \mathfrak{v} \rrbracket=$ $\llbracket Q(\mathfrak{v}), \mathfrak{u} \rrbracket$ for any $\mathfrak{u}, \mathfrak{v} \in \mathbb{H}(\Sigma)$. Thanks to these remarks, the second property is obtained by direct calculus from $(14)$. Finally $\operatorname{Ker}(\operatorname{Id}-\widetilde{\Pi})=\mathbb{X}(\Sigma)$ according to Proposition 5.3, which can be rewritten $\mathfrak{u} \in \mathbb{X}(\Sigma) \Longleftrightarrow \mathfrak{u}=\widetilde{\Pi}(\mathfrak{u})$.

\section{Quasi-local multi-trace formulation}

The operator $\widetilde{\Pi}$ satisfies the same elementary properties as $\Pi$. It involves non-local contributions but maps continuously $\mathbb{H}(\Sigma)$ onto itself. As a consequence we can derive a new (so called "quasi-local") multi-trace formulation from it, mimicking the derivation of the local multi-trace formulation presented in [17].

Assume that $\mathfrak{u}=\left(\gamma^{j}(u)\right)_{j=0}^{n}$ are the traces of the unique solution $u$ to Problem (1). Then the wave equations in each subdomain can be rewritten $(A-I d)\left(\mathfrak{u}-\mathfrak{u}_{\text {inc }}\right)=0$, and the transmission conditions can be written $\mathfrak{u}=\widetilde{\Pi}(\mathfrak{u})$. So it satisfies

$$
\left\{\begin{array}{l}
\mathfrak{u} \in \mathbb{H}(\Sigma) \text { and } \\
\llbracket(\mathrm{A}-\widetilde{\Pi}) \mathfrak{u}, \mathfrak{v} \rrbracket=\llbracket \mathfrak{f}, \mathfrak{v} \rrbracket \quad \forall \mathfrak{v} \in \mathbb{H}(\Sigma) .
\end{array}\right.
$$

where $\mathfrak{f}:=(A-I d) \mathfrak{u}_{\text {inc }}$. A striking point of the preceding construction is that it leads to a (generalised) Garding inequality for the formulation above.

\section{Theorem 6.1.}

Define $\Theta: \mathbb{H}(\Sigma) \rightarrow \mathbb{H}(\Sigma)$ by $\Theta(\mathfrak{u})=\left(-u_{j}, p_{j}\right)_{j=0}^{n}$ for any $\mathfrak{u}=\left(u_{j}, p_{j}\right) \in \mathbb{H}(\Sigma)$. Then there exists a constant $C>0$ and a compact operator $\mathrm{R}: \mathbb{H}(\Sigma) \rightarrow \mathbb{H}(\Sigma)$ such that

$$
\Re e\{\llbracket(\mathrm{A}-\widetilde{\Pi}+\mathrm{R}) \mathfrak{u}, \Theta(\overline{\mathfrak{u}}) \rrbracket\} \geq C\|\mathfrak{u}\|_{\mathbb{H}(\Sigma)}^{2} \quad \forall \mathfrak{u} \in \mathbb{H}(\Sigma) .
$$

\section{Proof:}


We already know that such an inequality is satisfied by A, see $[23, \S 4.1]$ for example. Thus it suffices to study $\mathfrak{u} \mapsto \Re e\{\llbracket \widetilde{\Pi}(\mathfrak{u}), \Theta(\overline{\mathfrak{u}}) \rrbracket\}$. Note that $\psi \Theta(\mathfrak{v})=\Theta(\psi \mathfrak{v})$ and $\chi \Theta(\mathfrak{v})=\Theta(\chi \mathfrak{v})$ for any $\mathfrak{v} \in \mathbb{H}(\Sigma)$. Applying the definition of $\widetilde{\Pi}$ yields

$$
\llbracket \widetilde{\Pi}(\mathfrak{u}), \Theta(\overline{\mathfrak{u}}) \rrbracket=\llbracket \chi \Pi(\mathfrak{u}), \Theta(\overline{\mathfrak{u}}) \rrbracket+\llbracket Q(\mathfrak{v}), \Theta(\overline{\mathfrak{v}}) \rrbracket .
$$

with $\mathfrak{v}=\psi \mathfrak{u}$. Elementary calculus on the definition (8) of $\Pi$ shows that $\Theta \Pi=\Pi \Theta$. Moreover we have $\llbracket \Theta(\mathfrak{w}), \mathfrak{w} \rrbracket=-\llbracket \mathfrak{w}, \Theta(\mathfrak{w}) \rrbracket$. So we obtain $\llbracket \chi \Pi(\mathfrak{u}), \Theta(\overline{\mathfrak{u}}) \rrbracket=\llbracket \chi \Pi(\Theta \overline{\mathfrak{u}}), \mathfrak{u} \rrbracket=$ $\llbracket \Theta(\chi \Pi(\overline{\mathfrak{u}})), \mathfrak{u} \rrbracket=-\overline{\llbracket \chi \Pi(\mathfrak{u}), \Theta(\overline{\mathfrak{u}}) \rrbracket} . \quad$ As a consequence $\Re e\{\llbracket \chi \Pi(\mathfrak{u}), \Theta(\overline{\mathfrak{u}}) \rrbracket\}=0$. Using this to evaluate the real part of (16), and according to Proposition 5.2, we see that there exists a compact operator $\mathrm{R}^{\prime}: \mathbb{H}(\Sigma) \rightarrow \mathbb{H}(\Sigma)$ such that $\Re e\left\{\llbracket\left(-\widetilde{\Pi}+\mathrm{R}^{\prime}\right) \mathfrak{u}, \Theta(\overline{\mathfrak{u}}) \rrbracket\right\} \geq 0$ which concludes that proof.

As a first consequence of the previous result, the operator associated to the quasi-local multitrace formulation (15) is Fredholm of index 0. In particular, it is an isomorphism if and only if it is one-to-one. We now prove injectivity.

\section{Theorem 6.2.}

Assume that the kernel $\mathscr{K}$ coming into play in (11) is real valued. Then, for $\kappa_{0}, \ldots, \kappa_{n}$ taking any real values, the operator $\mathrm{A}-\widetilde{\Pi}: \mathbb{H}(\Sigma) \rightarrow \mathbb{H}(\Sigma)$ is a continuous isomorphism.

\section{Proof:}

As mentioned before, we only need to prove injectivity. So take any $\mathfrak{u}=\left(\mathfrak{u}_{j}\right)_{j=0}^{n} \in \mathbb{H}(\Sigma)$ satisfying $(\mathrm{A}-\widetilde{\Pi}) \mathfrak{u}=0$. Thus we have $\mathfrak{w}:=(\widetilde{\Pi}+\mathrm{Id}) \mathfrak{u}=(\mathrm{A}+\mathrm{Id}) \mathfrak{u} \in \operatorname{range}(\mathrm{A}+\mathrm{Id}) \cap \mathbb{X}(\Sigma)$. Since $\mathcal{C}^{\text {in }}(\Sigma)=$ range $(\mathrm{A}+\mathrm{Id})$ and $\mathcal{C}^{\text {in }}(\Sigma) \cap \mathbb{X}(\Sigma)=\{0\}$ according to Lemma 3.2, we conclude that $\mathfrak{w}=0$. Set $\psi_{j}(\boldsymbol{x}):=G_{\kappa_{j}}^{j}\left(\mathfrak{u}_{j}\right)(\boldsymbol{x})$. We have $(\mathrm{Id}+\mathrm{A}) \mathfrak{u}=0$ which implies $\psi_{j}(\boldsymbol{x})=0$ for $\boldsymbol{x} \in \Omega_{j}$. So if we can prove that $\psi_{j}(\boldsymbol{x})=0$ for $\boldsymbol{x} \in \mathbb{R}^{d} \backslash \bar{\Omega}_{j}$, this will show that $\left[\gamma^{j}\left(\psi_{j}\right)\right]=$ $\left[\gamma^{j}\right] \cdot G_{\kappa_{j}}^{j}\left(\mathfrak{u}_{j}\right)=\mathfrak{u}_{j}=0$.

Since the kernel $\mathscr{K}$ coming into play in (11) is real valued, we have $\overline{\widetilde{\Pi}(\mathfrak{u})}=\widetilde{\Pi}(\overline{\mathfrak{u}})$. In addition we have $\widetilde{\Pi}^{2}=\operatorname{Id}$ and $\widetilde{\Pi}(\mathfrak{u})=-\mathfrak{u}$. As a consequence $2 \mathfrak{u}=\mathfrak{u}-\widetilde{\Pi}(\mathfrak{u})$, and $\overline{\mathfrak{u}}+\widetilde{\Pi}(\overline{\mathfrak{u}})=0$. From this and $(9)$, we obtain $2 \llbracket \mathfrak{u}, \overline{\mathfrak{u}} \rrbracket=\llbracket \mathfrak{u}-\widetilde{\Pi}(\mathfrak{u}), \overline{\mathfrak{u}} \rrbracket=-\llbracket \overline{\mathfrak{u}}+\widetilde{\Pi}(\overline{\mathfrak{u}}), \mathfrak{u} \rrbracket=0$. Since $\mathfrak{u}_{j}=\left[\gamma^{j}\right] \cdot G_{\kappa_{j}}^{j}\left(\mathfrak{u}_{j}\right)=$ $-\gamma_{c}^{j} \cdot G_{\kappa_{j}}^{j}\left(\mathfrak{u}_{j}\right)$, this can be rewritten

$$
\sum_{j=0}^{n} \Im m\left\{\int_{\Gamma_{j}} \gamma_{\mathrm{D}, c}^{j}\left(\psi_{j}\right) \gamma_{\mathrm{N}, c}^{j}\left(\bar{\psi}_{j}\right) d \sigma\right\}=0
$$

Take $r>0$ sufficiently large to guarantee that $\mathbb{R}^{d} \backslash \Omega_{0} \subset \mathrm{B}_{r}$ where $\mathrm{B}_{r} \subset \mathbb{R}^{d}$ refers to the ball centred at 0 with radius $r$. Since $-\Delta \psi_{j}-\kappa_{j}^{2} \psi_{j}=0$ in $\mathbb{R}^{d} \backslash \bar{\Omega}_{j}$, applying Green's formula in each $\mathrm{B}_{r} \backslash \bar{\Omega}_{j}$ yields

$$
\begin{aligned}
\int_{\partial \mathrm{B}_{r}} \psi_{j} \partial_{r} \bar{\psi}_{j} d \sigma & =\int_{\mathrm{B}_{r} \backslash \Omega_{j}}\left|\nabla \psi_{j}\right|^{2}-\kappa_{j}^{2}\left|\psi_{j}\right|^{2} d \boldsymbol{x}+\int_{\partial \Omega_{j}} \gamma_{\mathrm{D}, c}^{j}\left(\psi_{j}\right) \gamma_{\mathrm{N}, c}^{j}\left(\bar{\psi}_{j}\right) d \sigma \\
0 & =\int_{\mathrm{B}_{r} \backslash \Omega_{0}}\left|\nabla \psi_{0}\right|^{2}-\kappa_{0}^{2}\left|\psi_{0}\right|^{2} d \boldsymbol{x}+\int_{\partial \Omega_{0}} \gamma_{\mathrm{D}, c}^{0}\left(\psi_{0}\right) \gamma_{\mathrm{N}, c}^{0}\left(\bar{\psi}_{0}\right) d \sigma
\end{aligned}
$$

In the equations above " $\partial_{r}$ " refer to the radial derivative. Take the imaginary part of the identities above, and sum over $j=0 \ldots n$, taking account of (17). This leads to

$$
\sum_{j=0}^{n} \Im m\left\{\int_{\partial \mathrm{B}_{r}} \psi_{j} \partial_{r} \bar{\psi}_{j} d \sigma\right\}=\Im m\left\{\sum_{j=0}^{n} \int_{\partial \Omega_{j}} \gamma_{\mathrm{D}, c}^{j}\left(\psi_{j}\right) \gamma_{\mathrm{N}, c}^{j}\left(\bar{\psi}_{j}\right) d \sigma\right\}=\frac{1}{2 \mathfrak{\imath}} \llbracket \mathfrak{u}, \overline{\mathfrak{u}} \rrbracket=0
$$


where $\boldsymbol{\imath}$ refers to the imaginary unit. By construction, the functions $\psi_{j}$ are outgoing radiating, so that $0=\lim _{r \rightarrow \infty} \int_{\partial \mathrm{B}_{r}}\left|\partial_{r} \psi_{j}-\boldsymbol{\imath} \kappa_{j} \psi_{j}\right|^{2} d \sigma=0$. As a consequence, we finally obtain

$$
\begin{aligned}
& \sum_{j=0}^{n} \frac{1}{\kappa_{j}} \int_{\partial \mathrm{B}_{r}}\left|\partial_{r} \psi_{j}\right|^{2}+\kappa_{j}^{2}\left|\psi_{j}\right|^{2} d \sigma \\
& \quad=\sum_{j=1}^{n} \frac{1}{\kappa_{j}} \int_{\partial \mathrm{B}_{r}}\left|\partial_{r} \psi_{j}-\boldsymbol{\imath} \kappa_{j} \psi_{j}\right|^{2} d \sigma-2 \sum_{j=1}^{n} \Im m\left\{\int_{\partial \mathrm{B}_{r}} \psi_{j} \partial_{r} \bar{\psi}_{j} d \sigma\right\} \\
& =\sum_{j=1}^{n} \frac{1}{\kappa_{j}} \int_{\partial \mathrm{B}_{r}}\left|\partial_{r} \psi_{j}-\imath \kappa_{j} \psi_{j}\right|^{2} d \sigma \underset{r \rightarrow \infty}{\longrightarrow} 0
\end{aligned}
$$

This shows in particular that $\lim _{r \rightarrow \infty} \int_{\partial \mathrm{B}_{r}}\left|\psi_{j}\right|^{2} d \sigma=0$ for all $j=1 \ldots n$. As a consequence we can apply Rellich's lemma, see [12, Lemma 3.11], which implies that $\psi_{j}=0$ in the unbounded connected component of each $\mathbb{R}^{d} \backslash \bar{\Omega}_{j}$.

Let us prove that $\psi_{j}$ also vanishes in bounded connected components of $\mathbb{R}^{d} \backslash \bar{\Omega}_{j}$. First of all we have $\mathfrak{u}=-\widetilde{\Pi}(\mathfrak{u})$. Besides, according to the definition $(14)$ of $\widetilde{\Pi}$, we have $\widetilde{\Pi}(\mathfrak{v})=\Pi(\mathfrak{v})$ for all $\mathfrak{v} \in \mathbb{H}(\Sigma)$ supported on $\Sigma \backslash \mathscr{V}_{2 \epsilon}$. Since $\mathfrak{u}_{j}=-\gamma_{c}^{j}\left(\psi_{j}\right)$, this implies in particular

$$
\begin{aligned}
& \gamma_{\mathrm{D}, c}^{j}\left(\psi_{j}\right)=-\gamma_{\mathrm{D}, c}^{k}\left(\psi_{k}\right) \\
& \gamma_{\mathrm{N}, c}^{j}\left(\psi_{j}\right)=\gamma_{\mathrm{N}, c}^{k}\left(\psi_{k}\right) \quad \text { on } \Gamma_{j, k} \backslash \mathscr{V}_{2 \epsilon} \neq \emptyset
\end{aligned}
$$

Take an arbitrary $j$, and let $\mathcal{O}$ be a bounded connected component of $\mathbb{R}^{d} \backslash \bar{\Omega}_{j}$. We have $\partial \Omega_{j} \cap \partial \Omega_{k}=\Gamma_{j, k} \subset \partial \mathcal{O}$ for some $k=0 \ldots n, k \neq j$. Let $\mathcal{O}^{\prime}$ be the unbounded connected component of $\mathbb{R}^{d} \backslash \bar{\Omega}_{k}$. Then we have $\Omega_{j} \subset \mathcal{O}^{\prime}$, and $\partial \mathcal{O}^{\prime} \cap \partial \mathcal{O}=\partial \Omega_{j} \cap \partial \Omega_{k}=\Gamma_{j, k}$. Since $\psi_{k}=0$ in $\mathcal{O}^{\prime}$, we have $\left.\gamma_{\mathrm{D}, c}^{j}\left(\psi_{j}\right)\right|_{\Gamma_{j, k}}=-\gamma_{\mathrm{D}, c}^{k}\left(\psi_{k}\right)=0$ and $\left.\gamma_{\mathrm{N}, c}^{j}\left(\psi_{j}\right)\right|_{\Gamma_{j, k}}=\gamma_{\mathrm{N}, c}^{k}\left(\psi_{k}\right)=0$ on $\Gamma_{j, k} \backslash \mathscr{V}_{2 \epsilon} \neq \emptyset$ according to (18). Finally we have $-\Delta \psi_{j}-\kappa_{j}^{2} \psi_{j}=0$ in $\mathcal{O}$ with $\gamma_{c}^{j}\left(\psi_{j}\right)=0$ on $\Gamma_{j, k} \backslash \mathscr{V}_{2 \epsilon}$. We conclude by unique continuation principle (see [12, Thm.3.5]) that $\psi_{j}=0$ in $\mathcal{O}$. We have just proved that

$$
\psi_{j}=0 \quad \text { in } \mathbb{R}^{d} \backslash \bar{\Omega}_{j} \quad \forall j=0 \ldots n .
$$

Another important consequence of Theorem 6.1 concerns the solvability of Formulation (15) by means of a Galerkin approach: it guarantees a quasi-optimal convergence of the numerical solution toward the exact solution. Hence the following proposition is a direct application to Formulation (15) of Theorem 4.2.9 in [25].

\section{Proposition 6.1.}

Let $\left(\mathbb{H}_{h}(\Sigma)\right)_{0<h<1}$ be any dense sequence of finite dimensional subspaces in $\mathbb{H}(\Sigma)$ satisfying $\Theta\left(\mathbb{H}_{h}(\Sigma)\right) \subset \mathbb{H}_{h}(\Sigma)$. For any $\mathfrak{f} \in \mathbb{H}(\Sigma)$ there exists $h_{0}>0$ such that the following formulation admits a unique solution for any $h \in\left(0, h_{0}\right)$,

$$
\begin{aligned}
& \text { Find } \mathfrak{u}_{h} \in \mathbb{H}_{h}(\Sigma) \text { such that } \\
& \llbracket(\mathrm{A}-\widetilde{\Pi}) \mathfrak{u}_{h}, \mathfrak{v}_{h} \rrbracket=\llbracket \mathfrak{f}, \mathfrak{v}_{h} \rrbracket \quad \forall \mathfrak{v}_{h} \in \mathbb{H}_{h}(\Sigma)
\end{aligned}
$$

In addition there exists a constant $C>0$ independent of $h$ such that, if $\mathfrak{u} \in \mathbb{H}(\Sigma)$ refers to the unique solution to (15), we have

$$
\left\|\mathfrak{u}-\mathfrak{u}_{h}\right\|_{\mathbb{H}(\Sigma)} \leq C \inf _{\mathfrak{v}_{h} \in \mathbb{H}_{h}(\Sigma)}\left\|\mathfrak{u}-\mathfrak{v}_{h}\right\|_{\mathbb{H}(\Sigma)} \quad \forall h \in\left(0, h_{0}\right) .
$$


To conclude this section, since the operator $\mathrm{A}-\widetilde{\Pi} \operatorname{maps} \mathbb{H}(\Sigma)$ onto itself, it perfectly fits the operator preconditioning methodology described in [16], provided that the Galerkin discretisation under consideration induces stable discrete inf-sup conditions for the duality pairing $\llbracket \cdot, \cdot \rrbracket$ i.e. satisfies assumption $(2.3)$ in $[16]$.

\section{Numerical experiments}

This section is dedicated to describing numerical results related to the quasi-local multi-trace formulation (15). We consider a 2-D problem with a scatterer corresponding to the geometry depicted in the figure below. This situation is similar to the one considered in $[8,11,17]$, which makes comparisons with the results of these references easier.
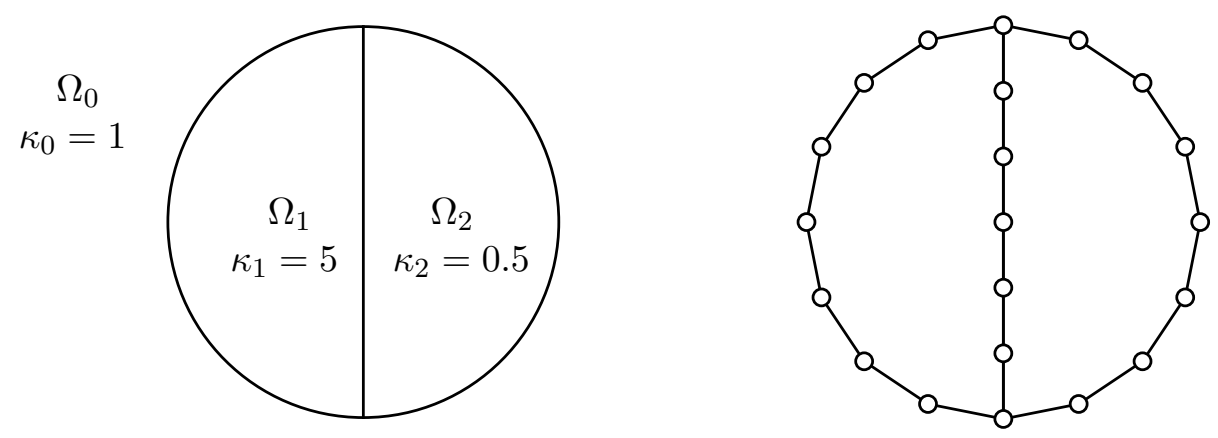

Figure 2: Geometry considered for the numerical experiments and corresponding meshes

With this geometry, we consider Problem (1) with the incident field $u_{\text {inc }}(\boldsymbol{x})=\exp \left(\boldsymbol{\imath} \kappa_{0} \mathbf{e}_{1} \cdot \boldsymbol{x}\right)$, and the wave numbers $\kappa_{0}=1, \kappa_{1}=5, \kappa_{2}=0.5$. As regards discretisation, we consider a uniform panelling $\Sigma^{h} \simeq \Sigma$ which induces a mesh for each of the subdomains $\Gamma_{j}^{h} \simeq \Gamma_{j}, \Gamma_{j}^{h} \subset \Sigma^{h}$. The discrete spaces $\mathbb{H}_{h}(\Sigma)$ are constructed on these meshes by means of $\mathbb{P}_{1}$-Lagrange shape functions for both Dirichlet and Neumann traces

$$
\begin{aligned}
\mathbb{H}_{h}(\Sigma)=\{ & \left(u_{j}^{h}, p_{j}^{h}\right)_{j=0,1,2} \text { such that } \\
& \left.\forall j=0,1,2, \text { for all panel } e \subset \Gamma_{j}^{h},\left.u_{j}^{h}\right|_{e},\left.p_{j}^{h}\right|_{e} \in \mathbb{P}_{1}(e)\right\} .
\end{aligned}
$$

To obtain a reference solution in this experiment, we rely on the Poggio-Miller-ChangHarrington-Wu-Tsai (PMCHWT) formulation. This is a widespread formulation (see [3, $15,20,27])$ that is known to yield quasi-optimal convergence rate under conforming Galerkin discretisations, which justifies considering it for obtaining reference discrete solution. In the present situation (continuous) PMCHWT formulation writes

$$
\text { Find } \mathfrak{u} \in \mathbb{X}(\Sigma), \quad \llbracket A \mathfrak{u}, \mathfrak{v} \rrbracket=\llbracket \mathfrak{f}, \mathfrak{v} \rrbracket \quad \forall \mathfrak{v} \in \mathbb{X}(\Sigma) .
$$

We refer the reader to $[8,23]$ for further details on the analysis of PMCHWT. The results presented in this section will then involve the discrete solutions to three different boundary integral formulations namely

- $\mathfrak{u}_{h}^{\mathrm{L}}$ solves the local multi-trace formulation (10)

- $\mathfrak{u}_{h}^{\mathrm{QL}}$ solves the quasi-local multi-trace formulation (19)

- $\mathfrak{u}_{h}^{\mathrm{STF}}$ solves the PMCHWT formulation (21) 
All computations have been achieved on a laptop equipped with a 2-core Intel i7-3520M processor at $2.9 \mathrm{GHz}$ with $4 \mathrm{~GB}$ of RAM. Meshes have been generated using Gmsh [14] (see also the website http://geuz.org/gmsh/) and, for linear algebra including GMRes routines, we relied on the Gmm++ library (see http://download.gna.org/getfem/html/homepage/gmm/ index.html). When assembling the matrix associated to the transmission operator $\widetilde{\Pi}$ we considered the following choice of cut-off function,

$$
\begin{aligned}
& \psi(\boldsymbol{x}):=\psi_{\alpha}\left(\boldsymbol{x}-\boldsymbol{x}_{+}\right)+\psi_{\alpha}\left(\boldsymbol{x}-\boldsymbol{x}_{-}\right) \\
\text {with } \quad \psi_{\alpha}(\boldsymbol{y}) & =\exp \left(-\alpha|\boldsymbol{y}|^{2}\right) \exp \left(\frac{1}{1-1 /(2|\boldsymbol{y}|)^{2}}\right) 1_{2|\boldsymbol{y}|<1}
\end{aligned}
$$

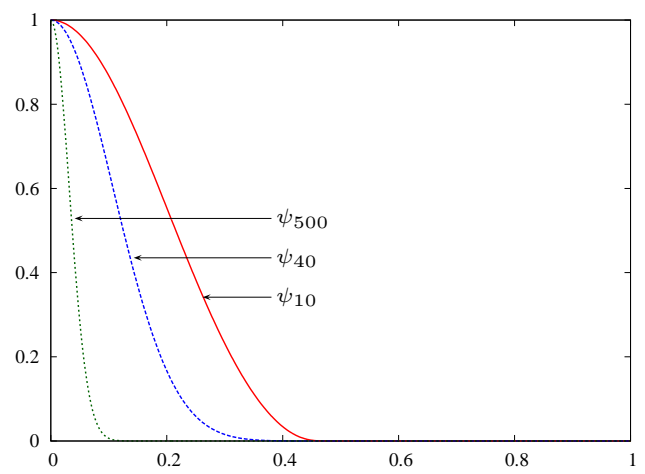

Figure 3: Shape of $\psi_{\alpha}$ for different values of $\alpha$

where $\boldsymbol{x}_{ \pm}:=(0, \pm 1)$ are the junction points, and $\alpha>0$ is some fitting parameter allowing to control how much the function $\psi$ is concentrated around junction points. For the first results we consider the value $\alpha=40$. As regards the kernel coming into play in (11), we chose the Laplace kernel

$$
\mathscr{K}(\boldsymbol{x})=\frac{1}{4 \pi|\boldsymbol{x}|}
$$

which guarantees that Theorem 6.2 holds. The table below presents the relative error between $\mathfrak{u}_{h}^{\mathrm{QL}}$ and $\mathfrak{u}_{h}^{\mathrm{STF}}$ on the one hand, and the relative error between $\mathfrak{u}_{h}^{\mathrm{L}}$ and $\mathfrak{u}_{h}^{\mathrm{STF}}$ on the other hand. These results are summarised graphically in Figure 4.

\begin{tabular}{ccc}
$\mathrm{h}$ & $\left\|\mathfrak{u}_{h}^{Q L}-\mathfrak{u}_{h}^{S T F}\right\|_{\mathbb{H}}$ & $\left\|\mathfrak{u}_{h}^{L}-\mathfrak{u}_{h}^{S T F}\right\|_{\mathbb{H}}$ \\
\hline 0.0981353 & 0.00563797 & 0.010623 \\
\hline 0.0490825 & 0.00127879 & 0.00237613 \\
\hline 0.0253347 & 0.000310327 & 0.000574992 \\
\hline 0.00994171 & $4.77015 \mathrm{e}-05$ & $8.70664 \mathrm{e}-05$ \\
\hline 0.00500253 & $1.18548 \mathrm{e}-05$ & $2.15064 \mathrm{e}-05$
\end{tabular}

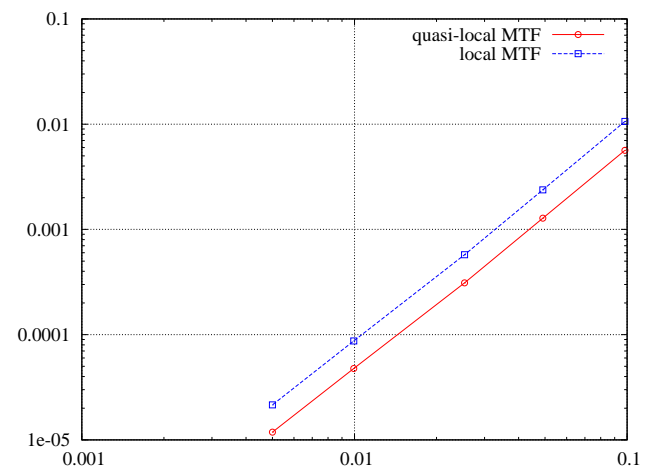

Figure 4: Errors of solutions to local/quasi-local formulation with respect to the PMCHWT solution versus $h=$ meshwidth 
These results show that $\mathfrak{u}_{h}^{\mathrm{L}}$ and $\mathfrak{u}_{h}^{\mathrm{QL}}$ approximate $\mathfrak{u}_{h}^{\mathrm{STF}}$ with a comparable level of consistency, although the approximation provided by the quasi-local formulation seems slightly more precise (about twice more precise for the same mesh width).

Denote $\mathrm{M}_{h}, \mathrm{~B}_{h}^{\mathrm{L}}, \mathrm{B}_{h}^{\mathrm{QL}}$ the matrices obtain by Galerkin discretisation of the bilinear forms $\llbracket \cdot, \cdot \rrbracket$, $\llbracket(\mathrm{A}-\Pi) \cdot, \cdot \rrbracket, \llbracket(\mathrm{A}-\widetilde{\Pi}) \cdot, \cdot \rrbracket$. Figure 5 below represents the spectrum of the matrices $\mathrm{M}_{h}^{-1} \mathrm{~B}_{h}^{\mathrm{L}}$ and $\mathrm{M}_{h}^{-1} \mathrm{~B}_{h}^{\mathrm{QL}}$ that may be considered as approximations of the spectrum of the continuous operators $\mathrm{A}-\Pi$ and $\mathrm{A}-\widetilde{\Pi}$ (see e.g. [1] for further details on the numerical analysis of eigenvalue problems in the context Galerkin discretisation). The eigenvalues have been computed by means of the Arpack++ library (see http://www.caam.rice.edu/software/ARPACK/ arpack++.html), using a mesh with 167 nodes corresponding to 672 unknowns for both local and quasi-local multi-trace discrete formulations. In both cases, eigenvalues clearly cluster in two packets symmetric to each other with respect to the origin.
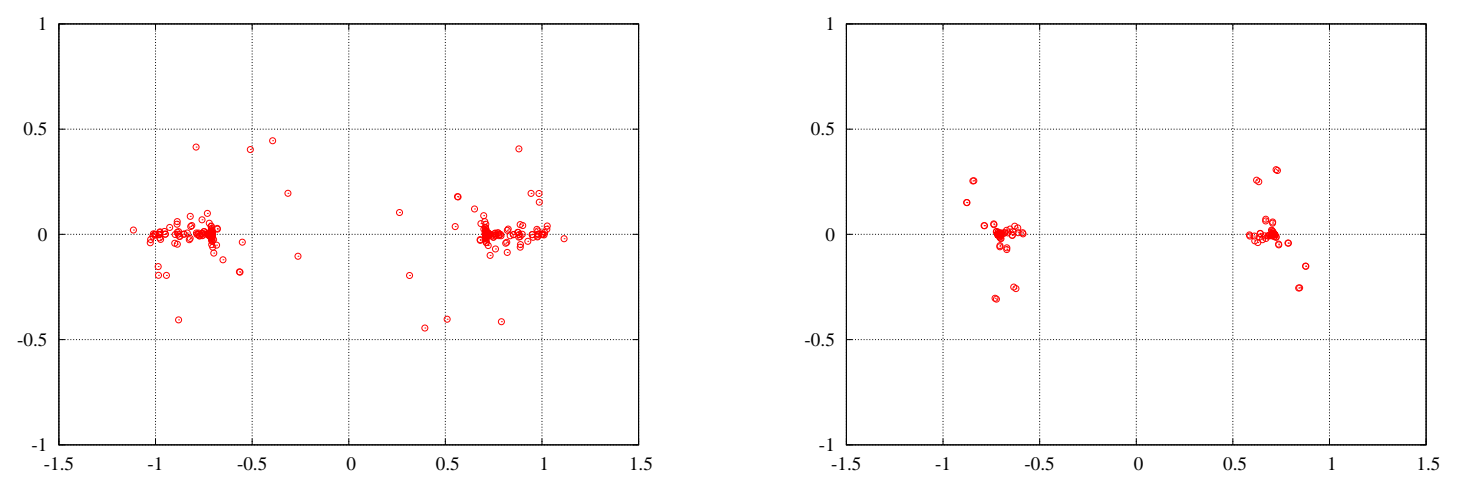

Figure 5: Spectrum of the operator $\mathrm{M}_{h}^{-1} \mathrm{~B}_{h}^{\mathrm{QL}}$ (on the left) and $\mathrm{M}_{h}^{-1} \mathrm{~B}_{h}^{\mathrm{L}}$ (on the right).

In Figure 6 we have represented the convergence history of GMRes iterations when applied to the matrices $\mathrm{M}_{h}^{-1} \mathrm{~B}_{h}^{\mathrm{QL}}$ and $\mathrm{M}_{h}^{-1} \mathrm{~B}_{h}^{\mathrm{L}}$ corresponding respectively to quasi-local and local multitrace. We used a restart of 20 iterations and a threshold of $10^{-8}$. For a complete description of the GMRes iterative solver see e.g. [24]. Convergence of GMRes is stable with respect to the mesh width in both cases, and both formulations converge at a comparable speed.
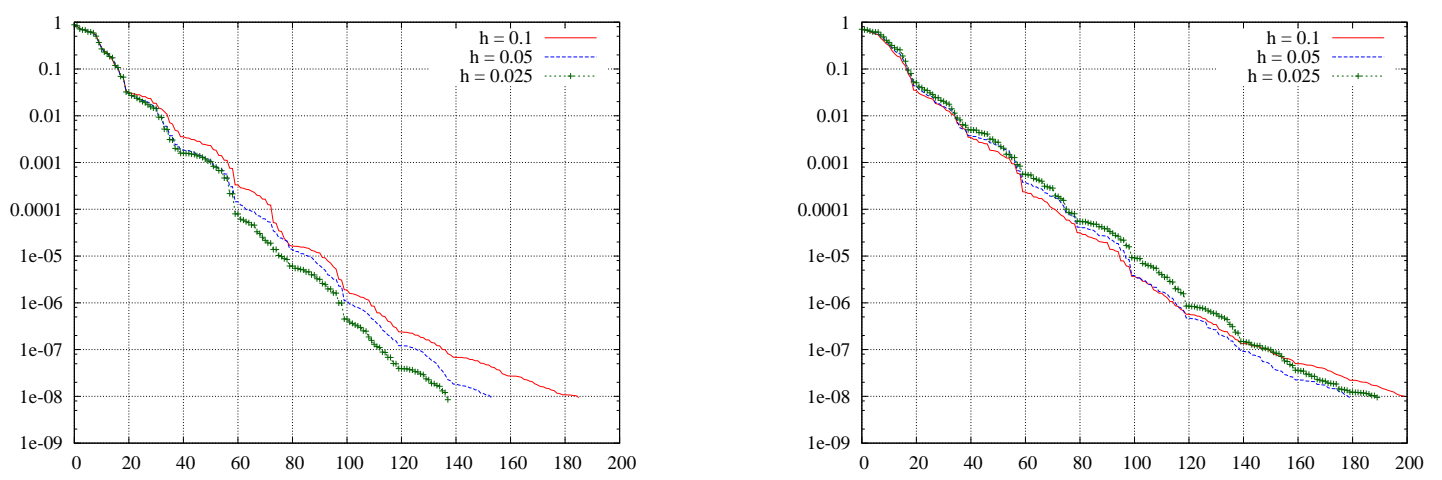

Figure 6: Convergence history of GMRes iterations (norm of the residual vs. number of iterations) for quasi-local multi-trace (left) and local multi-trace (right) for three values of the mesh width. 
It is natural to ask about the influence of the choice of the cut-off function $\psi$ on the performances of the quasi-local multi-trace formulation. Let us first examine the impact of the parameter $\alpha$ coming into play in Formula (22) on the consistency. In the table below, we compare the errors $\left\|\mathfrak{u}_{h}^{Q L}-\mathfrak{u}_{h}^{S T F}\right\|_{\mathbb{H}}$ associated to different values of the mesh width and for $\alpha=10,40,100,1000$. The value of this parameter seems to have very little impact on the accuracy.

\begin{tabular}{c|c|c|c|c} 
& \multicolumn{4}{|c}{ Error $\left\|\mathfrak{u}_{h}^{Q L}-\mathfrak{u}_{h}^{S T F}\right\|_{\mathbb{H}}$ with } \\
$\mathrm{h}$ & $\alpha=10$ & $\alpha=40$ & $\alpha=100$ & $\alpha=1000$ \\
\hline 0.0981353 & 0.00572272 & 0.00563797 & 0.0054629 & 0.00505463 \\
\hline 0.0490825 & 0.00128214 & 0.00127879 & 0.00127138 & 0.00115966 \\
\hline 0.0253347 & 0.000310538 & 0.000310327 & 0.000309988 & 0.000303461 \\
\hline 0.00994171 & $4.77078 \mathrm{e}-05$ & $4.77015 \mathrm{e}-05$ & $4.7693 \mathrm{e}-05$ & $4.75972 \mathrm{e}-05$ \\
\hline 0.00500253 & $1.18553 \mathrm{e}-05$ & $1.18548 \mathrm{e}-05$ & $1.18541 \mathrm{e}-05$ & $1.18484 \mathrm{e}-05$
\end{tabular}

Finally, in Figure 7 below we examine the impact of a variation of the parameter $\alpha$ on the convergence of GMRes solver (with a restart of 20 iterations) applied to quasi-local multitrace formulation at a fixed mesh width $h=0.05$. We can see that convergence deteriorates as $\alpha \rightarrow 0$.

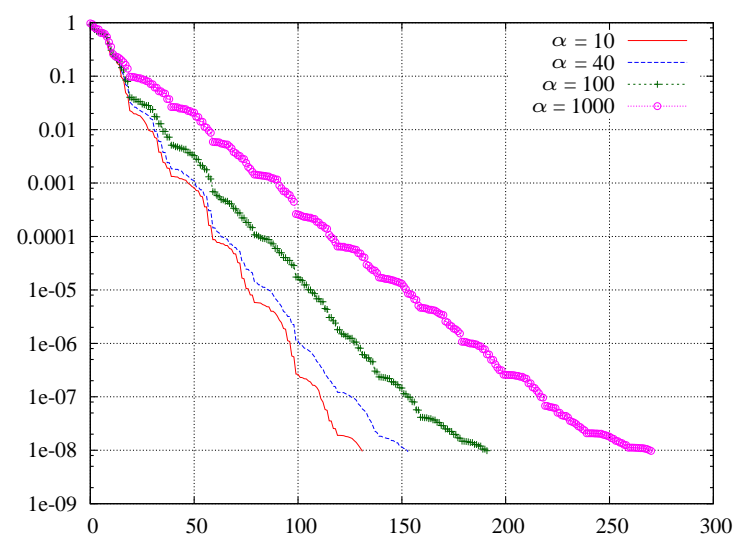

Figure 7: Convergence history of GMRes iterations (norm of the residual vs. number of iterations) for quasi-local multi-trace with $h=0.05$ and $\alpha=10,40,100,1000$.

\section{References}

[1] D. Boffi. Finite element approximation of eigenvalue problems. Acta Numerica, 19:1-120, 52010.

[2] A. Buffa. Remarks on the discretization of some noncoercive operator with applications to the heterogeneous Maxwell equations. SIAM J. Numer. Anal., 43(1):1-18, 2005. 
[3] Y. Chang and R. Harrington. A surface formulation or characteristic modes of material bodies. IEEE Trans. Antennas and Propagation, 25:789-795, 1977.

[4] S.H. Christiansen and J-C. Nédélec. Des préconditionneurs pour la résolution numérique des équations intégrales de frontière de l'acoustique. C. R. Acad. Sci. Paris Sér. I Math., 330(7):617-622, 2000.

[5] S.H. Christiansen and J-C. Nédélec. A preconditioner for the electric field integral equation based on Calderon formulas. SIAM J. Numer. Anal., 40(3):1100-1135, 2002.

[6] X. Claeys. A single trace integral formulation of the second kind for acoustic scattering. Technical Report no. 2011-14, SAM, ETH Zürich, 2011.

[7] X. Claeys and R. Hiptmair. Integral equations on multi-screens. Integral Equations Operator Theory, 77(2):167-197, 2013.

[8] X. Claeys and R. Hiptmair. Multi-trace boundary integral formulation for acoustic scattering by composite structures. Comm. Pure Appl. Math., 66(8):1163-1201, 2013.

[9] X. Claeys and R. Hiptmair. Integral equations for acoustic scattering by partially impenetrable composite objects. Technical Report 2014-06, Seminar for Applied Mathematics, ETH Zürich, Switzerland, 2014.

[10] X. Claeys, R. Hiptmair, and C. Jerez-Hanckes. Multi-trace boundary integral equations. In Direct and Inverse Problems in Wave Propagation and Applications. I. Graham, U. Langer, M. Sini, M. Melenk, 2012.

[11] X. Claeys, R. Hiptmair, and E. Spindler. A second-kind galerkin boundary element method for scattering at composite objects. Technical Report 2013-13 (revised), Seminar for Applied Mathematics, ETH Zürich, Switzerland, 2013.

[12] D. Colton and R. Kress. Integral equation methods in scattering theory. Pure and Applied Mathematics (New York). John Wiley \& Sons Inc., New York, 1983. A Wiley-Interscience Publication.

[13] M. Costabel. Boundary integral operators on Lipschitz domains: elementary results. SIAM J. Math. Anal., 19(3):613-626, 1988.

[14] C. Geuzaine and J.-F. Remacle. Gmsh: A 3-D finite element mesh generator with builtin pre- and post-processing facilities. Internat. J. Numer. Methods Engrg., 79(11):13091331, 2009.

[15] R. Harrington. Boundary integral formulations for homogeneous material bodies. $J$. Electromagnetic Waves and Applications, 3(1):1-15, 1989.

[16] R. Hiptmair. Operator preconditioning. Comput. Math. Appl., 52(5):699-706, 2006.

[17] R. Hiptmair and C. Jerez-Hanckes. Multiple traces boundary integral formulation for Helmholtz transmission problems. Adv. Comput. Math., 37(1):39-91, 2012.

[18] U. Langer and O. Steinbach. Boundary Element Tearing and Interconnecting Methods. Computing, 71:205-228, 2003. 
[19] W. McLean. Strongly elliptic systems and boundary integral equations. Cambridge University Press, Cambridge, 2000.

[20] E. Miller and A. Poggio. Computer Techniques for Electromagnetics, chapter Integral equation solution of three-dimensional scattering problems, chapter 4, pages 159-263. Pergamon, New York, 1973.

[21] G. Of and O. Steinbach. The all-floating boundary element tearing and interconnecting method. J. Numer. Math., 17(4):277-298, 2009.

[22] G. Of, O. Steinbach, and W. L. Wendland. Boundary element tearing and interconnecting domain decomposition methods. In Multifield problems in solid and fluid mechanics, volume 28 of Lect. Notes Appl. Comput. Mech., pages 461-490. Springer, Berlin, 2006.

[23] T. von Petersdorff. Boundary Integral Equations for Mixed Dirichlet, Neumann and Transmission Problems. Math. Met. App. Sc., 11:185-213, 1989.

[24] Y. Saad. Iterative methods for sparse linear systems. Society for Industrial and Applied Mathematics, Philadelphia, PA, second edition, 2003.

[25] S.A. Sauter and C. Schwab. Boundary element methods, volume 39 of Springer Series in Computational Mathematics. Springer-Verlag, Berlin, 2011.

[26] O. Steinbach and W. L. Wendland. The construction of some efficient preconditioners in the boundary element method. Adv. Comput. Math., 9(1-2):191-216, 1998. Numerical treatment of boundary integral equations.

[27] T.-K. Wu and L. L. Tsai. Scattering from arbitrarily-shaped lossy dielectric bodies of revolution. Radio Science, 12(5):709-718, 1977. 